\title{
Multicolour deep CCD photometric study of the moderately young southern open star clusters NGC 3228, NGC 4103, NGC 5662 and NGC 6087
}

\author{
R. Sagar ${ }^{1,2, \star \star}$ and R.D. Cannon ${ }^{2}$ \\ 1 Indian Institute of Astrophysics, Bangalore 560034, India \\ e-mail address: sagar@upso.ernet.in \\ 2 Anglo-Australian Observatory, P.O. Box 296, Epping NSW 2121, Australia \\ e-mail address: rdc@aaoepp.aao.gov.au
}

Received April 2; accepted June 26, 1996

\begin{abstract}
CCD observations in the $U, B, \quad V, R$ and $I$ passbands have been used to generate colourcolour and deep colour-magnitude diagrams for the young open star clusters NGC 3228, NGC 4103, NGC 5662 and NGC 6087. The sample consists of about 2000 stars reaching down to $V=20 \mathrm{mag}$. The interstellar extinction across the face of the imaged cluster regions seems to be uniform with average values of $E(B-V)=$ $0.31,0.34$ and $0.22 \mathrm{mag}$ for NGC 4103, NGC 5662 and NGC 6087 respectively. The distances to the clusters NGC 3228, NGC 4103, NGC 5662 and NGC 6087 have been estimated as $0.5 \pm 0.05,2.0 \pm 0.3,0.8 \pm 0.1$ and $1.0 \pm 0.1 \mathrm{kpc}$ respectively, while the corresponding ages estimated from the theoretical stellar evolutionary isochrones fitted to the bright proper motion cluster members are $\sim 100,30,80$ and $65 \mathrm{Myr}$ respectively. Present data are consistent with the membership of the Cepheid variable V Cen to NGC 5662 and of S Norm to NGC 6087. Heavy contamination by foreground and background field stars has unfortunately made it impossible to reach definite conclusions about the faint luminosity function, and the data do not reach faint enough limits to investigate pre-main sequence stars.
\end{abstract}

Key words: HR diagram - stars: evolution - open clusters and associations: individual: NGC 3228, NGC 4103, NGC 5662, NGC 6087

Send offprint requests to: R. Sagar

* Tables 6 to 9 only available in electronic form at CDS.

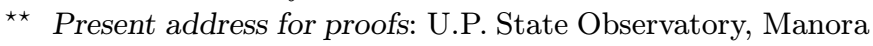
Peak, Naini Tal - 263129 (UP) India.

\section{Introduction}

The colour magnitude diagrams (CMDs) of moderately young $(\sim 20-50 \mathrm{Myr})$ open star clusters show the effect of stellar evolution at the brighter end and contain pre-main sequence (PMS) stars at the fainter end. Consequently, they provide a rare opportunity for reliable estimation of ages of high and low mass stars formed in a molecular cloud. So they can be used to determine the length of time over which star formation was active in a particular region and also to know which stars form first - high mass or low mass ones or if they formed together.

In order to address the questions raised above, we performed faint $(V \sim 16-18 \mathrm{mag})$ multicolour CCD stellar photometry in five southern moderately young open star clusters, namely NGC 3228, NGC 4103, NGC 4755, NGC 5662 and NGC 6087. Based on these observations, the presence of PMS stars in NGC 4755 has been indicated for the first time by Sagar \& Cannon (1995). They also found that massive $\left(M>10 M_{\odot}\right)$ and low mass $\left(M<2 M_{\odot}\right)$ stars in the cluster are formed nearly at the same time from a molecular cloud which might have existed for a period of about $6-7 \mathrm{Myr}$. UBVRI photometry and similar analyses for the other four clusters are presented here.

Table 1 lists the relevant prior information (taken from Lyngà's (1987) catalogue) available on the clusters under discussion. Some of these parameters should probably be revised to take account of the new data presented here. The clusters all lie close to the galactic plane $\left(|b|<6^{\circ}\right.$ and $|Z| \leq 85 \mathrm{pc})$ with moderate interstellar extinction $(E(B-V) \leq 0.3 \mathrm{mag})$ and at a galactocentric distance of $\sim 8 \mathrm{kpc}$. Their ages and heliocentric distances range from $\sim 40$ to $130 \mathrm{Myr}$ and 0.5 to $1.5 \mathrm{kpc}$ respectively. The clusters under discussion are relatively compact objects with angular radii $\leq 6^{\prime}$. NGC 5662 and NGC 6087 each have one Cepheid variable. NGC 4103 contains an interesting 
Table 1. Preliminary informations about the clusters under study. They are taken from the catalogue of Lyngå (1987)

\begin{tabular}{|c|c|c|c|c|c|c|c|c|c|c|c|}
\hline \multicolumn{3}{|c|}{ Cluster designation } & \multicolumn{4}{|c|}{ Galactic } & \multirow{2}{*}{$\begin{array}{l}\text { Trumpler } \\
\text { class }\end{array}$} & \multirow{2}{*}{$\begin{array}{l}\text { Radius } \\
\left({ }^{\prime}\right)\end{array}$} & \multirow{2}{*}{$\begin{array}{l}\text { distance } \\
\text { (pc) }\end{array}$} & \multirow{2}{*}{$\begin{array}{c}E(B-V) \\
(\mathrm{mag})\end{array}$} & \multirow{2}{*}{$\begin{array}{l}\text { age } \\
\text { (Myr) }\end{array}$} \\
\hline NGC & IAU & OCL & $\begin{array}{c}l \\
\left({ }^{\circ}\right)\end{array}$ & $\begin{array}{c}b \\
\left({ }^{\circ}\right)\end{array}$ & $\begin{array}{c}R \\
(\mathrm{pc})\end{array}$ & $\begin{array}{c}Z \\
(\mathrm{pc})\end{array}$ & & & & & \\
\hline 3228 & C1019-514 & 800 & 280.74 & 4.58 & 8420 & 39 & II3p & 2.5 & 500 & 0.03 & 63 \\
\hline 4103 & C1204-609 & 871 & 297.57 & 1.17 & 7920 & 29 & $\mathrm{I} 2 \mathrm{~m}$ & 2.5 & 1500 & 0.32 & 40 \\
\hline 5662 & C1431-563 & 928 & 316.90 & 3.47 & 8050 & 37 & II3r & 6 & 600 & 0.31 & 63 \\
\hline 6087 & C1614-577 & 948 & 327.76 & -5.40 & 7750 & -85 & $\mathrm{II} 2 \mathrm{~m}$ & 6 & 900 & 0.18 & 63 \\
\hline
\end{tabular}

eclipsing variable. All the clusters contain chemically peculiar stars which may be due to their youthfulness.

Previous studies, the new observations and data reductions are described in the next three sections. The interstellar extinction, other photometric results and their implications for star formation processes are described in subsequent sections of this paper.

\section{Previous studies}

In order to separate bright $(V \leq 13.3 \mathrm{mag})$ cluster members from field stars, kinematical (mainly proper motion) studies have been carried out in all cluster regions except NGC 3228. Relative proper motions have been determined in the regions of NGC 4103, NGC 5662 and NGC 6087 by King (1979, 1980 and 1982 respectively) and the probability of cluster membership for individual stars has also been derived. Other details of the proper motion studies are given in Table 2. Using these proper motion data, Sagar \& Bhatt (1989a,b) have studied the internal kinematics in NGC 4103, NGC 5662 and NGC 6087 and found that within the errors of measurement intrinsic dispersions are independent of stellar mass and radial distance.

Table 2. Details of the proper motion studies carried out by King (1979), (1980) and (1982) for NGC 4103, NGC 5662 and NGC 6087 respectively. Relative proper motions have been determined for $N_{\mathrm{t}}$ stars. Out of them $N_{\mathrm{m}}$ are liekly cluster members. $\sigma_{\mu x}$ and $\sigma_{\mu y}$ are the orthogonal components of the intrinsic proper motion dispersion determined by Sagar \& Bhatt $(1989 a, b)$

\begin{tabular}{lccccc}
\hline Cluster & $\begin{array}{c}\text { Accuracy } \\
\text { (')/century) }\end{array}$ & $N_{\mathrm{t}}$ & $N_{\mathrm{m}}$ & $\begin{array}{c}\sigma_{\mu x} \\
\left({ }^{\prime \prime} / \text { century }\right)\end{array}$ & $\begin{array}{c}\sigma_{\mu y} \\
\left({ }^{\prime \prime} / \text { century }\right)\end{array}$ \\
\hline NGC 4103 & 0.08 & 171 & 69 & $0.04 \pm 0.01$ & $0.06 \pm 0.01$ \\
NGC 5662 & 0.09 & 188 & 77 & $0.07 \pm 0.02$ & $0.11 \pm 0.02$ \\
NGC 6087 & 0.15 & 157 & 74 & $0.05 \pm 0.03$ & $0.09 \pm 0.02$ \\
\hline
\end{tabular}

A description of the earlier photometric and spectroscopic studies of the clusters under study is given below:

NGC 3228. An inconspicuous cluster located at a distance of $\sim 500 \mathrm{pc}$ on the inner edge of the CarinaCygnus arm of our Galaxy. This is the nearest but least studied cluster in our sample. The only detailed photometric study of the cluster is by Hogg (1963). He carried out photoelectric observations of 26 stars in the $U B V$ passbands. Only eleven of these are photometric members. The cluster suffers with very small interstellar extinction (see Table 1). Hartoog (1976) determined the spectral types for 13 stars, one of them an Am star.

NGC 4103. A moderately populous star cluster in Crux. Photoelectric $U B V$ photometry of 23 stars in the cluster region has been carried out by Pedreros (1984), while photographic $U B V$ photometry of 67 stars has been published by Becker et al. (1976). Wesselink (1969) studied the cluster extensively using $U B V$ photoelectric and $B V$ photographic photometry of 512 stars brighter than $V=17 \mathrm{mag}$ and spectral types of 12 brighter stars. These studies indicate that all brighter cluster members are dwarfs with spectral types later than B2, while there are no red giant members. Stetson (1981) carried out four colour and $\mathrm{H}_{\beta}$ photometry for 20 B-type stars in the cluster region. This study indicates that the cluster may contain an extreme Be star, perhaps with a circumstellar dust shell; there are also several stars with anomalously strong $\mathrm{H}_{\beta}$ absorption for their temperature and luminosities. All photometric studies indicate that interstellar extinction in front of the cluster is almost uniform, with a value of colour excess $E(B-V) \sim 0.32$ mag.

The semi-detached early-type (B2IV) eclipsing binary system AI Crucis (CPD - 60 3273 , COD - 60 3971 ) is the $5^{\text {th }}$ brightest member of the cluster sequence and has a proper motion cluster membership probability of 84\% (cf. King 1979). A detailed Strömgren photometric and medium-dispersion spectroscopic study of the object has been done by Bell et al. (1987). 
NGC 5662. This open star cluster is a loose concentration of late B- and A-type stars lying in Centaurus. Relatively few investigations on this cluster have been carried out so far. Moffat \& Vogt (1973) presented $U B V$ photoelectric photometry for 30 comparatively bright stars in the cluster area. Haug (1978) observed 28 stars photoelectrically and 280 stars photographically up to $V=15.5 \mathrm{mag}$ in the $U B V$ system. Recently, Clariá et al. (1991) presented $U B V$ photoelectric photometry for 237 stars brighter than $V=14$ mag as well as DDO and Washington photometry for three red stars. Strömgren photometry of 27 B- and A-type stars brighter than $V=11.5$ mag is presented by Schneider (1987). This and spectroscopic work by Fitzgerald et al. (1979) indicate that there are chemically peculiar stars in the cluster. All the photometric studies indicate that reddening across the cluster face is variable with a mean value of $E(B-V)=$ 0.31 mag. However, distance estimates to the cluster vary from 500 to 800 pc. The study by Clariá et al. (1991) indicates that the cluster contains two red giants and two photometric variables; it is $\sim 80 \mathrm{Myr}$ old and has a metal abundance of $[\mathrm{Fe} / \mathrm{H}]=-0.03 \pm 0.13$ relative to the Sun.

The probable association of the Cepheid variable $\mathrm{V}$ Centauri with the cluster has been discussed by Turner (1982) and more recently by Clariá et al. (1991). They conclude that, although the star V Cen lies at a distance slightly larger than two cluster radii from the cluster center, it is very likely a cluster member.

NGC 6087. This cluster lies near the southern boundary of the constellation Norma in an uniformly obscured region of the Sagittarius-Carina arm of the Galaxy. The Cepheid variable S Normae is situated near the optical center of the cluster and has a cluster proper motion membership probabilty of $99 \%$ (cf. King 1982). These data, as well as spectroscopic radial velocity measurements by Feast (1957) and more recently by Mermilliod et al. (1987), confirm the physical association of the Cepheid with the cluster.

Previous broadband $U B V$ photoelectric and/or photographic studies of NGC 6087 have been published by Irwin (1958), Fernie (1961), Landolt (1964), Breger (1966), Pedreros (1984) and Turner (1986). Graham (1967), Schmidt (1980) and Eggen (1980) carried out four colour and $\mathrm{H}_{\beta}$ photometry of 18,16 and 15 stars brighter than $V \sim 10 \mathrm{mag}$, respectively. All these studies indicate that reddening across the cluster is uniform with $E(B-V)=0.18 \mathrm{mag}$; distance estimates to the cluster range from 0.7 to $0.9 \mathrm{kpc}$ and the cluster seems to be $\sim 60 \mathrm{Myr}$ old. Recently, Luck (1994) has done an abundance analysis of three stars in the cluster region and found that $[\mathrm{Fe} / \mathrm{H}]=-0.01 \pm 0.23$ and $[\mathrm{O} / \mathrm{Fe}]=-0.33 \pm 0.11$.

The existing photometric studies of the star clusters under discussion are generally limited to $V \sim 15 \mathrm{mag}$, so that
PMS stellar evolutionary phases in the clusters could not be studied.

\section{Observations}

The observations in $U B V$ Johnson and $R I$ Kron - Cousins pass bands were carried out between 27 March - 2 April and 22 - 28 June 1989 on the ANU 1.0 metre telescope at Siding Spring Observatory, using a blue coated GEC P8603 CCD in an Astromed camera system mounted at the $\mathrm{f} / 8$ Cassegrain focus. During the observations typical seeing was 1.5 arcsec. The log of the observations is given in Table 3. Each $22 \mu \mathrm{m}$ square pixel of the $385 \times 578$ element CCD corresponds to 0.56 arcsec on the sky. The read-out noise for the system was about 8 electrons per pixel and the number of electrons per $\mathrm{ADU}$ is equal to 1. Flat-field exposures ranging from 1 to $5 \mathrm{~s}$ in each filter were made on the twilight sky. Graham's (1982) E-region standards (see Sagar \& Cannon 1995) were observed for calibration purposes. They cover a range in brightness $(8.8 \leq V \leq 16.5)$ as well as in colour $(0.03 \leq(V-I) \leq 1.73)$. The regions observed for the construction of the CMDs of the clusters are shown in Figs. 1-4 for NGC 3228, NGC 4103, NGC 5662 and NGC 6087 respectively. More than one region was imaged for each cluster, except for NGC 4103. The regions were chosen in a way to exclude the very brightest cluster members which would have saturated the CCD, but to include some previously photoelectrically observed stars and to be as near as possible to the cluster nucleus, thus maximising the number of measurable cluster members and minimising the proportion of field stars included in the CMD. The regions selected in this way are located generally well within the cluster diameter except in NGC 3228 where they are lying almost at edge of the cluster diameter and it turned out that we are too far from the cluster centre to catch any bright members or to see any faint ones clearly, so we are unable to add anything to previous data regarding its reddening, distance and age. The locations of the these regions with respect to each cluster centre are given in Table 4 . There is a small overlap between the two regions of NGC 3228 and NGC 5662 and between the regions 2 and 3 of NGC 6087 . To cover the widest possible range of magnitudes, generally 2 or 3 graded exposures in each pass band were taken (see Table 3 ). They range from 40 to $600 \mathrm{~s}$ in $U$, from 10 to $600 \mathrm{~s}$ in $B$ and from 1 to $300 \mathrm{~s}$ in $V, R$ and $I$.

\section{Reductions}

The data were reduced using computing facilities available at the Anglo-Australian Observatory, Epping and at the Indian Institute of Astrophysics, Bangalore. The flat-field and data CCD frames were bias subtracted and trimmed using the FIGARO software. The flat-field frames were 
Table 3. Log of CCD observations

\begin{tabular}{|c|c|c|c|c|}
\hline Cluster & Region & Filter & $\begin{array}{l}\text { Exposure time } \\
\text { (seconds) }\end{array}$ & Date \\
\hline \multirow[t]{2}{*}{ NGC 3228} & 1 & $\begin{array}{l}U \\
B \\
V \\
R \\
I\end{array}$ & $\begin{array}{l}100,600 \\
10,50,600 \\
10,300 \\
10,300 \\
10,300\end{array}$ & $\begin{array}{c}\text { 30/31 } \underset{\prime \prime}{\text { March } 1989} \\
\text { " } \\
\text { " } \\
\text { " }\end{array}$ \\
\hline & 2 & $\begin{array}{l}U \\
B \\
V \\
R \\
I\end{array}$ & $\begin{array}{l}100,600 \\
100,500 \\
5,20,60 \times 2,300 \times 3 \\
20,150 \\
10,150\end{array}$ & $\begin{array}{c}26 / 27 \underset{\prime \prime}{\text { June }} 1989 \\
\text { " } \\
\text { " } \\
\text { " }\end{array}$ \\
\hline NGC 4103 & 1 & $\begin{array}{l}U \\
B \\
V \\
R \\
I\end{array}$ & $\begin{array}{l}100,400 \\
40,300 \\
5,120 \\
10,100 \\
10,100\end{array}$ & $\begin{array}{c}26 / 27 \underset{\prime \prime}{\text { June }} 1989 \\
\text { ", } \\
\text { " } \\
\prime \prime\end{array}$ \\
\hline \multirow[t]{2}{*}{ NGC 5662} & 1 & $\begin{array}{l}U \\
B \\
V \\
R \\
I\end{array}$ & $\begin{array}{l}50,600 \\
50,600 \\
10,300 \\
10,100,300 \\
10,100,300\end{array}$ & $\begin{array}{c}28 / 29 \underset{\prime \prime}{\operatorname{March} 1989} \\
\prime \prime \\
\prime \prime \\
\prime \prime\end{array}$ \\
\hline & 2 & $\begin{array}{l}U \\
B \\
V \\
R \\
I\end{array}$ & $\begin{array}{l}40,100,400 \\
10,30,300 \\
5,200 \\
1,5,10,100 \\
3,60\end{array}$ & $\begin{array}{c}26 / 27 \underset{\prime \prime}{\operatorname{June}} 1989 \\
" \prime \\
\prime \prime \\
\prime \prime\end{array}$ \\
\hline \multirow[t]{3}{*}{ NGC 6087} & 1 & $\begin{array}{l}U \\
B \\
V \\
I\end{array}$ & $\begin{array}{l}100,600 \\
30,300 \\
10,300 \\
10,300\end{array}$ & $\begin{array}{c}27 / 28 \underset{\prime \prime}{\text { March } 1989} \\
\text { " } \\
\prime \prime\end{array}$ \\
\hline & 2 & $\begin{array}{l}U \\
B \\
V \\
R \\
I\end{array}$ & $\begin{array}{l}100 \\
30 \\
10 \\
20 \\
10\end{array}$ & $\begin{array}{c}27 / 28 \underset{\prime \prime}{\operatorname{March} 1989} \\
\text { " } \\
\prime \prime \\
\prime \prime\end{array}$ \\
\hline & 3 & $\begin{array}{l}U \\
B \\
V \\
R \\
I\end{array}$ & $\begin{array}{l}100,600 \\
100,600 \\
50,200 \\
10,100 \\
10,100\end{array}$ & $\begin{array}{c}25 / 26 \text { June } 1989 \\
\text { "I } \\
\text { " } \\
\text { " }\end{array}$ \\
\hline
\end{tabular}

Table 4. Relative positions of the imaged regions from the cluster centre

\begin{tabular}{|c|c|c|c|c|c|c|c|c|}
\hline \multirow[t]{3}{*}{ Cluster } & \multicolumn{6}{|c|}{ Central coordinates for 1950} & \multirow[t]{3}{*}{ Region } & \multirow[t]{3}{*}{ Location } \\
\hline & & $\alpha$ & & & $\delta$ & & & \\
\hline & (h) & $(\mathrm{m})$ & (s) & $\left({ }^{\circ}\right)$ & $\left({ }^{\prime}\right)$ & $\left({ }^{\prime \prime}\right)$ & & \\
\hline NGC 3228 & 10 & 19 & 21.7 & -51 & 29 & 20 & $\begin{array}{l}1 \\
2\end{array}$ & $\begin{array}{l}\sim 6^{\prime} \text { south } \\
\sim 4^{\prime} \text { south }\end{array}$ \\
\hline NGC 4103 & 12 & 04 & 06.3 & -60 & 57 & 20 & 1 & $\sim 5^{\prime}$ west \\
\hline NGC 5662 & 14 & 32 & 23.3 & -56 & 19 & 43 & $\begin{array}{l}1 \\
2\end{array}$ & $\begin{array}{l}\sim 1^{\prime} \text { south } \\
\sim 4^{\prime} \text { south }\end{array}$ \\
\hline NGC 6087 & 16 & 14 & 40.2 & -57 & 47 & 14 & $\begin{array}{l}1 \\
2 \\
3\end{array}$ & $\begin{array}{l}\sim 2^{\prime} \text {, north } \\
\sim 2^{\prime} \text { south } \\
\sim 4^{\prime} \text { south-west }\end{array}$ \\
\hline
\end{tabular}


summed for each colour band. The evenness of flat fields is better than a few percent in all the filters.

The magnitude estimate of a star on the data frames has been done using DAOPHOT software (Stetson 1987, 1992). Further processing and conversion of these raw instrumental magnitudes into the standard photometric system have been done using the procedure outlined by Stetson (1992). The image parameters and errors provided by DAOPHOT were used to reject poor measurements. About $10 \%$ of the stars were rejected in this process. The DAOMASTER program was used for cross identifying the stars measured on different frames of a cluster region. In those cases where brighter stars are saturated on deep exposure frames, their magnitudes have been taken only from the short exposure frames. Most of the stars brighter than $V \sim 10.5$ mag could not be measured because they are saturated even on the shortest exposure frames. The magnitudes of fainter stars have been taken only from the deep exposure frames as they are either not detected or have very small $S / N$ ratio on short exposure frames.

The CCD instrumental magnitudes have been calibrated using the colour equations given by Sagar \& Cannon (1995) for NGC 4755, as the present observations were carried out with the same equipment during the same period. For evaluating the zero-points for the data frames, we have used the mean values of atmospheric extinction for the site. For establishing the local standards, we selected about 30 isolated stars in each field and used the DAOGROW program for construction of the aperture growth curve required for determining the difference between aperture and profile-fitting magnitudes. These differences, together with the differences in exposure times and atmospheric extinction, were used in evaluating zeropoints for local standards in the cluster frames. The zeropoints are uncertain by $\sim 0.02 \mathrm{mag}$ in $U$ and $B$ and by $\sim 0.01 \mathrm{mag}$ in $V, R$ and $I$. The internal errors in the case of NGC 4103, estimated from the scatter in the individual measures on different exposures, are listed in Table 5 as a function of magnitude for each filter. The errors become large (more than $0.10 \mathrm{mag}$ ) for stars fainter than $V=17$. They can be considered as representative of the accuracy of our photometry in the other clusters.

The $X$ and $Y$ pixel coordinates as well as $V$, $(U-B),(B-V),(V-R)$ and $(V-I)$ magnitudes of the stars observed in NGC 3228, NGC 4103, NGC 5662 and NGC 6087 are listed in Tables $6,7,8$ and 9 respectively. They are generally average of at least two measurements. Stars observed by others have been identified in the last column. A few bright stars in each cluster are identified in the corresponding chart (see Figs. 1-4).

\section{Comparison with previous photometry}

In this section, we compare the present CCD photometry with all available previous photoelectric and photographic observations. We have found no relevant published

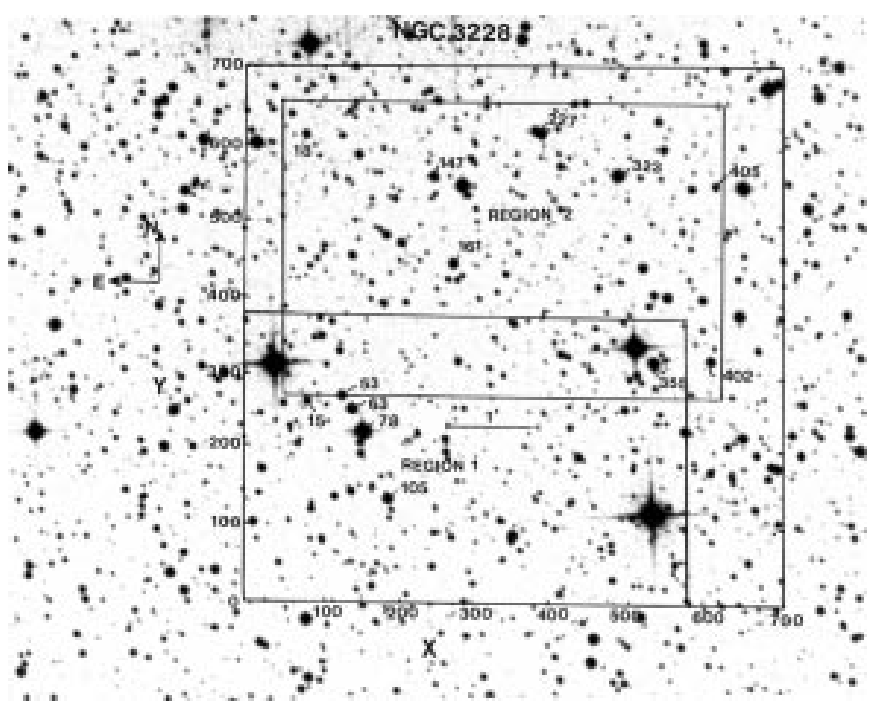

Fig. 1. Identification map for the two imaged regions of NGC 3228, reproduced from the ESO(B) sky survey. The size of a CCD frame is $3.5 \times 5.4$ arcmin and the coordinates are in pixel units. A few bright stars from Table 6 are identified

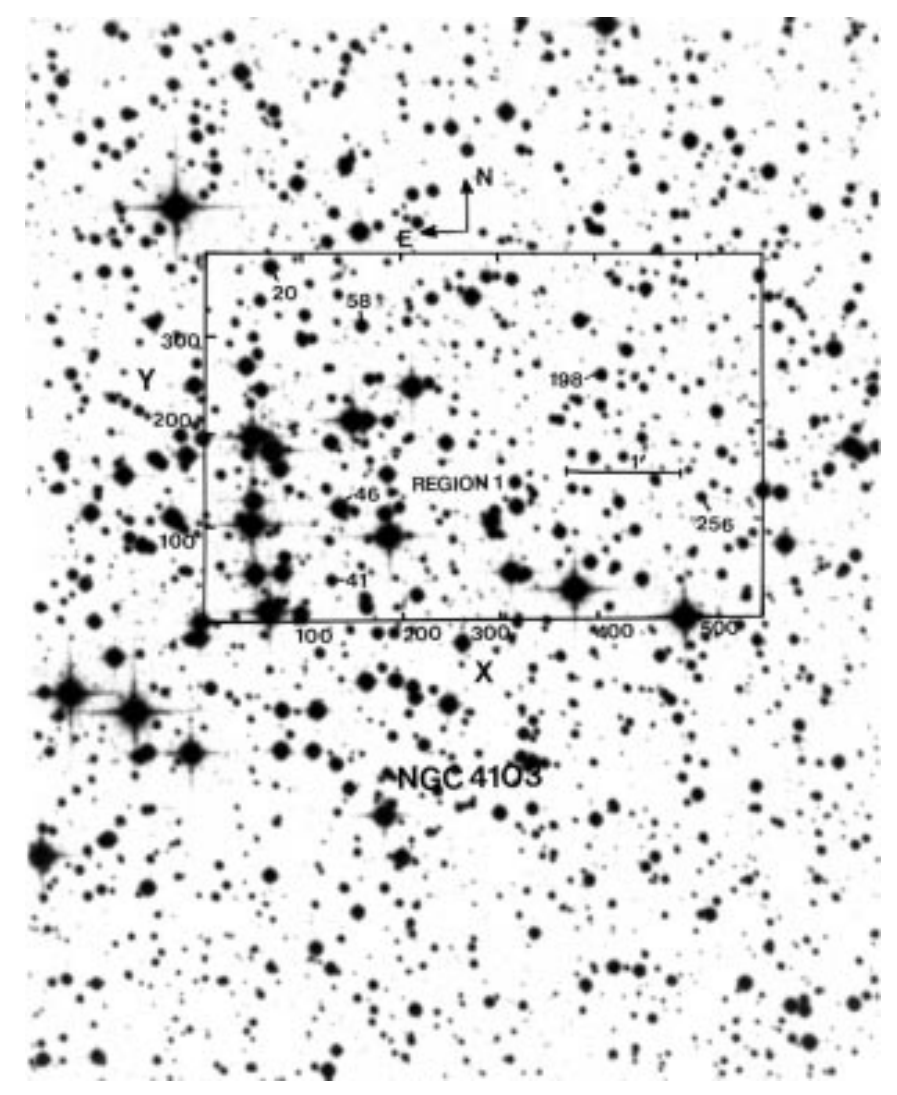

Fig. 2. Identification map for the imaged region of NGC 4103. Otherwise as for Fig. 1. A few bright stars from Table 7 are identified 
Table 10. Statistical results of the photometric comparison. The difference $(\Delta)$ is always in the sense present minus comparison data. The mean and standard deviation $(\sigma)$ are based on $\mathrm{N}$ stars. A few points discrepant by more than $3.5 \sigma$ have been excluded from the analysis

(A) NGC 4103. References Wesselink (1969), Pedreros (1984) and Becker et al. (1976) have been abbreviated as W, P and B respectively

\begin{tabular}{|c|c|c|c|c|}
\hline Comparison data & $V$ & $\begin{array}{c}\Delta V \\
\text { Mean } \pm \sigma\end{array}$ & $N$ & $\begin{array}{l}\Delta(B-V) \\
\text { Mean } \pm \sigma\end{array}$ \\
\hline CCD \& W photoelectric & $9.8-14.3$ & $-0.01 \pm 0.024$ & 12 & $0.008 \pm 0.020$ \\
\hline CCD \& P photoelectric & $9.8-13.8$ & $-0.044 \pm 0.044$ & 14 & $0.004 \pm 0.022$ \\
\hline W \& B photoelectric & $9.2-10.5$ & $0.068 \pm 0.031$ & 5 & $-0.020 \pm 0.016$ \\
\hline $\mathrm{W} \& \mathrm{P}$ photoelectric & $9.2-13.8$ & $-0.022 \pm 0.029$ & 12 & $-0.008 \pm 0.016$ \\
\hline W \& B photographic & $10.2-13.7$ & $0.188 \pm 0.074$ & 45 & $-0.111 \pm 0.081$ \\
\hline \multirow[t]{9}{*}{ CCD \& W photographic } & $10.2-13.0$ & $-0.052 \pm 0.050$ & 11 & $-0.009 \pm 0.052$ \\
\hline & $13.0-14.0$ & $-0.024 \pm 0.047$ & 9 & $0.037 \pm 0.055$ \\
\hline & $14.0-15.0$ & $0.002 \pm 0.049$ & 20 & $0.047 \pm 0.083$ \\
\hline & $15.0-15.5$ & $0.023 \pm 0.034$ & 11 & $-0.064 \pm 0.099$ \\
\hline & $15.5-16.0$ & $0.116 \pm 0.074$ & 15 & $-0.157 \pm 0.107$ \\
\hline & $16.0-16.25$ & $0.179 \pm 0.045$ & 10 & $-0.278 \pm 0.142$ \\
\hline & $16.25-16.5$ & $0.204 \pm 0.091$ & 14 & $-0.424 \pm 0.157$ \\
\hline & $16.5-16.75$ & $0.249 \pm 0.093$ & 15 & $-0.344 \pm 0.221$ \\
\hline & $16.75-17.2$ & $0.357 \pm 0.105$ & 9 & $-0.441 \pm 0.230$ \\
\hline \multirow{6}{*}{ CCD \& B photographic } & $9.7-11.7$ & $0.065 \pm 0.026$ & 6 & $-0.037 \pm 0.031$ \\
\hline & $11.7-12.5$ & $0.060 \pm 0.027$ & 6 & $-0.112 \pm 0.013$ \\
\hline & $12.5-13.3$ & $0.130 \pm 0.026$ & 5 & $-0.088 \pm 0.023$ \\
\hline & $13.3-14.0$ & $0.294 \pm 0.108$ & 5 & $-0.122 \pm 0.063$ \\
\hline & $B-V$ & $\Delta V$ & & $\Delta(B-V)$ \\
\hline & & Mean $\pm \sigma$ & $N$ & Mean $\pm \sigma$ \\
\hline \multirow[t]{6}{*}{ CCD \& W photographic } & $0.0-0.25$ & $-0.060 \pm 0.029$ & 12 & $0.003 \pm 0.036$ \\
\hline & $0.25-0.5$ & $0.031 \pm 0.125$ & 22 & $-0.078 \pm 0.331$ \\
\hline & $0.5-0.75$ & $0.106 \pm 0.142$ & 25 & $-0.176 \pm 0.232$ \\
\hline & $0.75-1.0$ & $0.184 \pm 0.077$ & 30 & $-0.276 \pm 0.147$ \\
\hline & $1.0-1.25$ & $0.232 \pm 0.148$ & 14 & $-0.218 \pm 0.141$ \\
\hline & $1.25-2.0$ & $0.076 \pm 0.093$ & 8 & $-0.125 \pm 0.145$ \\
\hline \multirow[t]{3}{*}{ CCD \& B photographic } & $0.0-0.1$ & $0.065 \pm 0.026$ & 6 & $-0.037 \pm 0.031$ \\
\hline & $0.1-0.15$ & $0.097 \pm 0.061$ & 10 & $-0.113 \pm 0.035$ \\
\hline & $0.25-0.4$ & $0.252 \pm 0.129$ & 6 & $-0.098 \pm 0.045$ \\
\hline
\end{tabular}

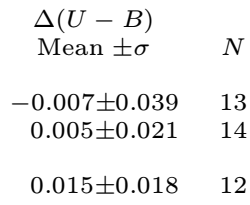

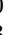

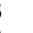

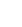

$N$
2
2
0
4
8
6
0
6

(B) NGC 5662. References Moffat \& Vogt (1973), Haug (1978) and Clariá et al. (1991) have been abbreviated as MV, H and C respectively

\section{Comparison data}

CCD \& MV photoelectric

CCD \& $\mathrm{H}$ photoelectric CCD \& C photoelectric CCD \& H photographic MV \& H photoelectric MV \& C photoelectric $\mathrm{H} \& \mathrm{C}$ photoelectric

$\mathrm{H}$ photographic \& $\mathrm{C}$ photoelectric

$\mathrm{H}$ photographic \& $\mathrm{H}$ photoelectric

\begin{tabular}{|c|c|c|}
\hline$\Delta V$ & & $\Delta(B-V)$ \\
\hline Mean $\pm \sigma$ & $N$ & Mean $\pm \sigma$ \\
\hline$-0.011 \pm 0.030$ & 8 & $0.003 \pm 0.034$ \\
\hline$-0.012 \pm 0.039$ & 9 & $-0.024 \pm 0.021$ \\
\hline$-0.060 \pm 0.046$ & 14 & $0.004 \pm 0.021$ \\
\hline$-0.023 \pm 0.040$ & 29 & $-0.011 \pm 0.040$ \\
\hline $0.009 \pm 0.017$ & 13 & $-0.022 \pm 0.020$ \\
\hline$-0.042 \pm 0.023$ & 28 & $0.002 \pm 0.019$ \\
\hline$-0.050 \pm 0.017$ & 19 & $0.026 \pm 0.017$ \\
\hline$-0.041 \pm 0.039$ & 76 & $0.019 \pm 0.032$ \\
\hline$-0.003 \pm 0.023$ & 26 & $-0.001 \pm 0.025$ \\
\hline
\end{tabular}

$-0.001 \pm 0.025$

$\begin{array}{ccc} & \Delta(U-B) & \\ N & \text { Mean } \pm \sigma & N \\ & & \\ 9 & -0.049 \pm 0.016 & 7 \\ 9 & -0.041 \pm 0.033 & 9 \\ 14 & -0.056 \pm 0.031 & 14 \\ 28 & -0.030 \pm 0.042 & 23 \\ 13 & -0.009 \pm 0.020 & 13 \\ 28 & 0.029 \pm 0.030 & 29 \\ 20 & 0.002 \pm 0.034 & 19 \\ 75 & 0.067 \pm 0.109 & 70 \\ 25 & -0.123 \pm 0.196 & 26\end{array}$

$-0.123 \pm 0.196 \quad 26$
(C) NGC 6087. References by Fernie (1961), Turner (1986), Breger (1966), Landolt (1964) and Pedreros (1984) have been abberviated as F, T, $\mathrm{B}, \mathrm{L}$ and $\mathrm{P}$ respectively

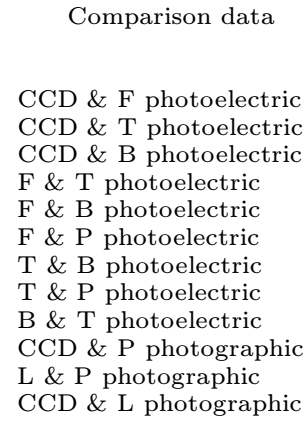

CCD \& L photographic

\begin{tabular}{cr}
\multicolumn{1}{c}{$V$} & \multicolumn{1}{c}{$\Delta V$} \\
& \multicolumn{1}{c}{ Mean $\pm \sigma$} \\
$10.0-12.5$ & $0.063 \pm 0.035$ \\
$10.0-12.6$ & $-0.032 \pm 0.026$ \\
$10.0-12.6$ & $-0.018 \pm 0.024$ \\
$8.2-12.2$ & $-0.057 \pm 0.060$ \\
$8.2-11.5$ & $-0.030 \pm 0.037$ \\
$8.2-11.5$ & $-0.022 \pm 0.037$ \\
$8.2-13.8$ & $-0.006 \pm 0.027$ \\
$8.2-13.8$ & $-0.008 \pm 0.013$ \\
$8.2-13.8$ & $0.008 \pm 0.021$ \\
$10.2-15.0$ & $-0.064 \pm 0.029$ \\
$10.0-15.2$ & $0.068 \pm 0.275$ \\
$10.0-13.0$ & $0.038 \pm 0.037$ \\
$13.0-14.0$ & $0.049 \pm 0.064$ \\
$14.0-14.5$ & $-0.009 \pm 0.065$ \\
$14.5-15.2$ & $-0.029 \pm 0.106$ \\
& \\
$B-V$ & \multicolumn{1}{c}{ Mean $\pm \sigma$} \\
& \\
$0.0-0.5$ & $0.036 \pm 0.045$ \\
$0.5-1.0$ & $0.123 \pm 0.125$ \\
$1.0-1.5$ & $0.015 \pm 0.039$ \\
$1.5-2.0$ & $0.124 \pm 0.110$
\end{tabular}

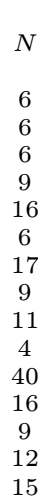

$\Delta(B-V)$

Mean $\pm \sigma$

$-0.006 \pm 0.036$ $0.035 \pm 0.035$

$0.027 \pm 0.034$

$0.041 \pm 0.020$

$0.036 \pm 0.014$

$0.013 \pm 0.020$

$0.004 \pm 0.014$

$-0.019 \pm 0.015$

$-0.022 \pm 0.018$

$-0.041 \pm 0.098$

$0.164 \pm 0.147$

$0.024 \pm 0.043$

$-0.044 \pm 0.039$

$0.079 \pm 0.124$

$0.139 \pm 0.117$

$\Delta(B-V)$

$N \quad$ Mean $\pm \sigma$

$0.013 \pm 0.049$

$0.020 \pm 0.115$

$0.044 \pm 0.088$

$-0.004 \pm 0.115$

\begin{tabular}{crc} 
& \multicolumn{1}{c}{$\Delta(U-B)$} \\
$N$ & \multicolumn{1}{c}{ Mean $\pm \sigma$} & $N$ \\
& & \\
6 & & \\
7 & $-0.031 \pm 0.040$ & 7 \\
6 & $0.012 \pm 0.022$ & 6 \\
10 & $-0.037 \pm 0.102$ & 4 \\
15 & $-0.004 \pm 0.060$ & 7 \\
10 & $0.045 \pm 0.067$ & 4 \\
17 & $0.042 \pm 0.025$ & 17 \\
9 & $0.068 \pm 0.017$ & 8 \\
11 & $0.055 \pm 0.098$ & 11 \\
4 & $-0.003 \pm 0.028$ & 4
\end{tabular}




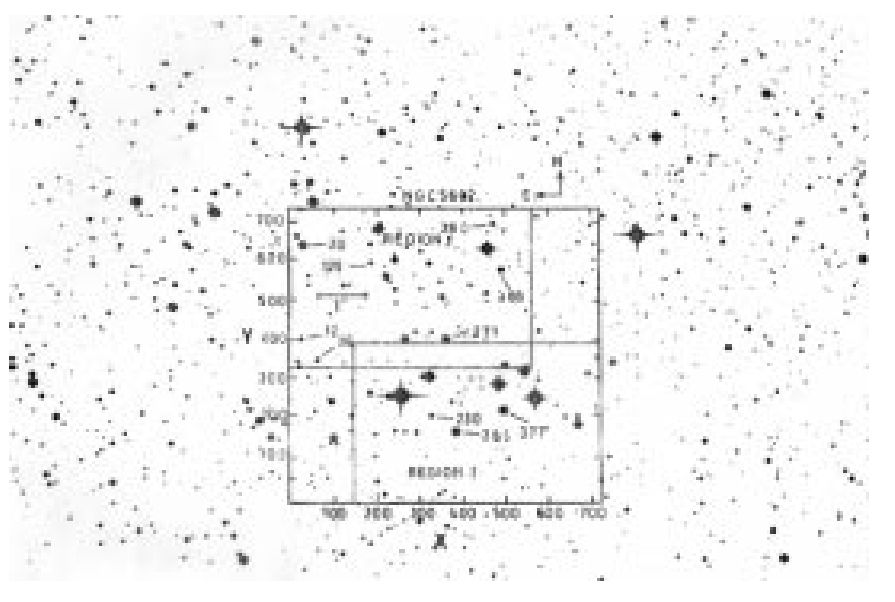

Fig. 3. Identification map for the two imaged regions of NGC 5662. Otherwise as for Fig. 1. A few bright stars from Table 8 are identified

a)

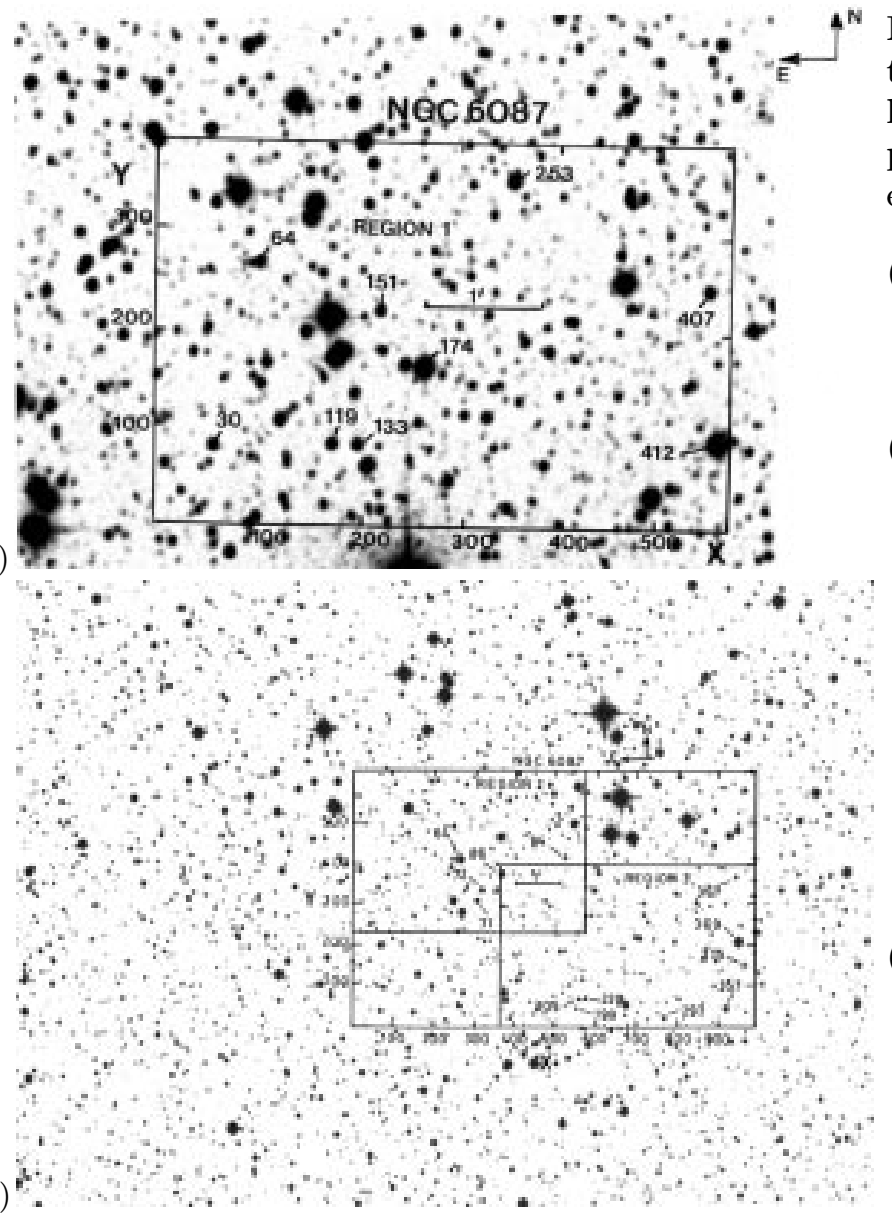

Fig. 4. Identification maps for NGC 6087 are given in a) and b) for the imaged regions 1 and 2, 3 respectively. Otherwise as for Fig. 1. A few bright stars from Table 9 are identified
Table 5. Internal photometric errors as a function of brightness. $\sigma$ is the standard deviation per observation in magnitude

$\begin{array}{cccccc}\text { Magnitude range } & \sigma_{U} & \sigma_{B} & \sigma_{V} & \sigma_{R} & \sigma_{I} \\ \leq 11.0 & 0.002 & 0.004 & 0.008 & 0.012 & 0.012 \\ 11.0-12.0 & 0.006 & 0.006 & 0.009 & 0.016 & 0.014 \\ 12.0-13.0 & 0.011 & 0.007 & 0.013 & 0.023 & 0.024 \\ 13.0-14.0 & 0.017 & 0.013 & 0.015 & 0.027 & 0.037 \\ 14.0-15.0 & 0.048 & 0.025 & 0.021 & 0.050 & 0.043 \\ 15.0-16.0 & 0.080 & 0.040 & 0.043 & 0.072 & 0.068 \\ 16.0-17.0 & 0.094 & 0.063 & 0.072 & 0.085 & 0.078 \\ 17.0-18.0 & - & 0.098 & 0.120 & - & 0.127\end{array}$

data for NGC 3228, while in the other three clusters the comparison is restricted to the $U, B$ and $V$ pass bands. The differences $(\Delta)$ between the present data and data obtained by others are plotted in Figs. 5, 6 and 7 for NGC 4103, NGC 5662 and NGC 6087 respectively, while the statistical results are given in Table 10. In the table we have also listed the results of intercomparing the various previous photoelectric and photographic data available for each cluster. Figures 5-7 and Table 10 show that:

(i) the $U B V$ photoelectric data obtained by different groups agree fairly well with each other. In contrast, the photographic data generally show large systematic differences compared with the photoelectric data.

(ii) in general the $U B V$ photoelectric photometric data are in fairly good agreement with the present CCD data. Except for a few outliers, the distribution of photometric differences seems to be random with no dependence on either brightness or colour (see Figs. 5$7)$. The largest discrepancies $(>0.3 \mathrm{mag}$ ), in each of $\Delta V, \Delta(B-V)$ and $\Delta(U-B)$, are for the star Haug 156 in NGC 5662, between our measurements and the data of Clariá et al. (1991). This seems to be due to misidentification by the latter observers since our data agree fairly well with the photographic measurements of the same star by Haug (1978).

(iii) the agreement between the $U B V$ photographic data and CCD data is satisfactory for NGC 5662 and also for the brighter $(V \leq 13 \mathrm{mag})$ stars in NGC 4103 and NGC 6087. However, the scatter is more than in the comparison of CCD with photoelectric data. For fainter stars in NGC 4103 and NGC 6087, the photographic data show systematically increasing differences with the CCD data in both $V$ magnitudes and $(B-V)$ colours. The differences become very large near the limiting magnitude of the photographic measurements. The errors are most likely to lie in the photographic observations since the CCD, unlike the photographic 
plate, is an intrinsically linear detector and the present data compares well with the photoelectric data over a wide range of brightness as well as colour.

\section{Interstellar extinction in the direction of the clusters}

In order to estimate interstellar extinction to the clusters, we have plotted apparent $(U-B)$ versus $(B-V)$ diagrams for all the stars measured by us in the clusters under study (Fig. 8). To identify the main sequence (MS) in these clusters, we have also plotted apparent $V,(B-V)$; $V,(U-B) ; V,(V-R)$ and $V,(V-I)$ diagrams for all the measured stars in Figs. 9, 10, 11 and 12 for NGC 3228, NGC 4103, NGC 5662 and NGC 6087 respectively. Adopting the slope of the reddening line $E(U-B) /$ $E(B-V)$ as 0.72 , we have fitted the intrinsic zero-age main-sequence (ZAMS) given by Schmidt-Kaler (1982) to the MS stars of spectral type earlier than A0 in Fig. 8. This yields a mean value of $E(B-V)=0.31,0.34$ and $0.22 \mathrm{mag}$ with an uncertainty of $\sim 0.03 \mathrm{mag}$ for NGC 4103, NGC 5662 and NGC 6087 respectively. The $E(B-V)$ value for NGC 3228 could not be estimated because of the lack of MS stars of spectral type earlier than A0 in our sample. This is evident from the cluster sequence present in the apparent $(U-B)$ versus $(B-V)$ diagram (Fig. 8a) and the CM diagrams in Fig. 9. So we adopt the value of $E(B-V)=0.03 \mathrm{mag}$ for this cluster from the work of Hogg (1963), based on much brighter stars. The observed cluster sequences in Fig. 8 are well-defined for the hotter stars, indicating that interstellar extinction is uniform across the face of the observed cluster regions of NGC 4103, NGC 5662 and NGC 6087. Our reddening estimates for the imaged regions agree fairly well with the values estimated earlier by others for the same regions (see Sect. 2). In the case of NGC 5662, Clariá et al. (1991) found variable interstellar extinction across the cluster face which is more than 10 times larger than the presently imaged area. However, their $E(B-V)$ value for the region imaged by $\mathrm{CCD}$ agrees fairly well with the present estimate.

For verifying the nature of the interstellar extinction law in the direction of each cluster, we used the stars having spectral type earlier than A0. This has been judged from their location in the $(U-B)$ versus $(B-V)$ and apparent $\mathrm{CM}$ diagrams (Figs. 9-12) which reveals that bright stars $(V<13 \mathrm{mag})$ with $(B-V)<0.35 \mathrm{mag}$ in NGC 4103; with $(B-V)<0.4$ mag in NGC 5662 and with $(B-V)<0.3 \mathrm{mag}$ in NGC 6087, are the desired objects. There are 23, 8 and 10 such stars in NGC 4103, NGC 5662 and NGC 6087 respectively. For these stars, the $(B-V)_{0}$, $E(B-V)$ and $E(U-B)$ values have been determined using either the spectral type (available only for 5 stars in NGC 5662 and 1 star in NGC 6087) taken from the Open
Cluster Data Base (cf. Mermilliod 1992), or the $U B V$ photometric $Q$ method (cf. Johnson \& Morgan 1953; Sagar \& Joshi 1979) and the calibration given by Schmidt-Kaler (1982). For calculating $E(V-R)$ and $E(V-I)$ values, we used the present $(V-R)$ and $(V-I)$ measurements, Sagar \& Cannon's (1994) calibration between $(B-V)_{0}$ and $(V-R)_{0}$ and Walker's (1985) calibration between $(B-V)_{0}$ and $(V-I)_{0}$. The mean values of the colour excess ratios derived in this way are listed in Table 11. They are in fair agreement with the normal values, indicating that the law of interstellar extinction in the direction of the clusters under discussion is normal.

\section{Distances to the clusters}

In order to determine the distance moduli of the clusters, we have plotted intrinsic $V_{0},(U-V)_{0} ; V_{0},(B-V)_{0} ; V_{0}$, $(V-R)_{0}$ and $V_{0},(V-I)_{0}$; diagrams for each cluster in Figs. 13, 14, 15 and 16. Stars (mostly proper motion and photometric cluster members) observed photoelectrically by others but not present in our sample have also been included in the plots, since the photoelectric data show good agreement with the present CCD measurements. They are taken from Hogg (1963) for NGC 3228, from Wesselink (1969) for NGC 4103, from Clariá et al. (1991) for NGC 5662 and from Fernie (1961), Breger (1966) and Turner (1986) for NGC 6087. In NGC 3228, we plotted the members assigned by Hogg (1963) using proper motion, photometric and spectroscopic data as there are no other membership studies available for the cluster. Proper motions for stars brighter than $B \sim 12$ mag have been derived in the regions of NGC 4103, NGC 5662 and NGC 6087 by King (1979, 1980 and 1982 respectively). Stars with photoelectric and/or CCD measurements but having proper motion cluster membership probability less than $50 \%$ have been excluded from the plots. In NGC 5662, Clariá's et al. (1991) photometric members not present in our sample have also been plotted in the CM diagram. In plotting these figures, we have converted apparent $V$ magnitudes and $(B-V),(U-B),(V-R)$ and $(V-I)$ colours into intrinsic ones using the values of $E(B-V)$ derived in Sect. 6 and the following relations for $E(U-B)$ (cf. Kamp 1974; Sagar \& Joshi 1979); $E(V-R)$ (cf. Alcalá \& Ferro 1988); $A_{v}$ and $E(V-I)$ (Walker 1987):

$E(U-B)=[X+0.05 E(B-V)] E(B-V)$

where $X=0.62-0.3(B-V)_{0}$ for $(B-V)_{0}<-0.09$ and $X=0.66+0.08(B-V)_{0}$ for $(B-V)_{0}>-0.09$;

$E(V-R)=[E 1+E 2 \times E(B-V)] E(B-V)$

where $E 1=0.6316+0.0713(B-V)_{0}$ and $E 2=0.0362+$ $0.0078(B-V)_{0}$;

$A_{v}=\left[3.06+0.25(B-V)_{0}+0.05 E(B-V)\right] E(B-V)$ 
NGC 4103
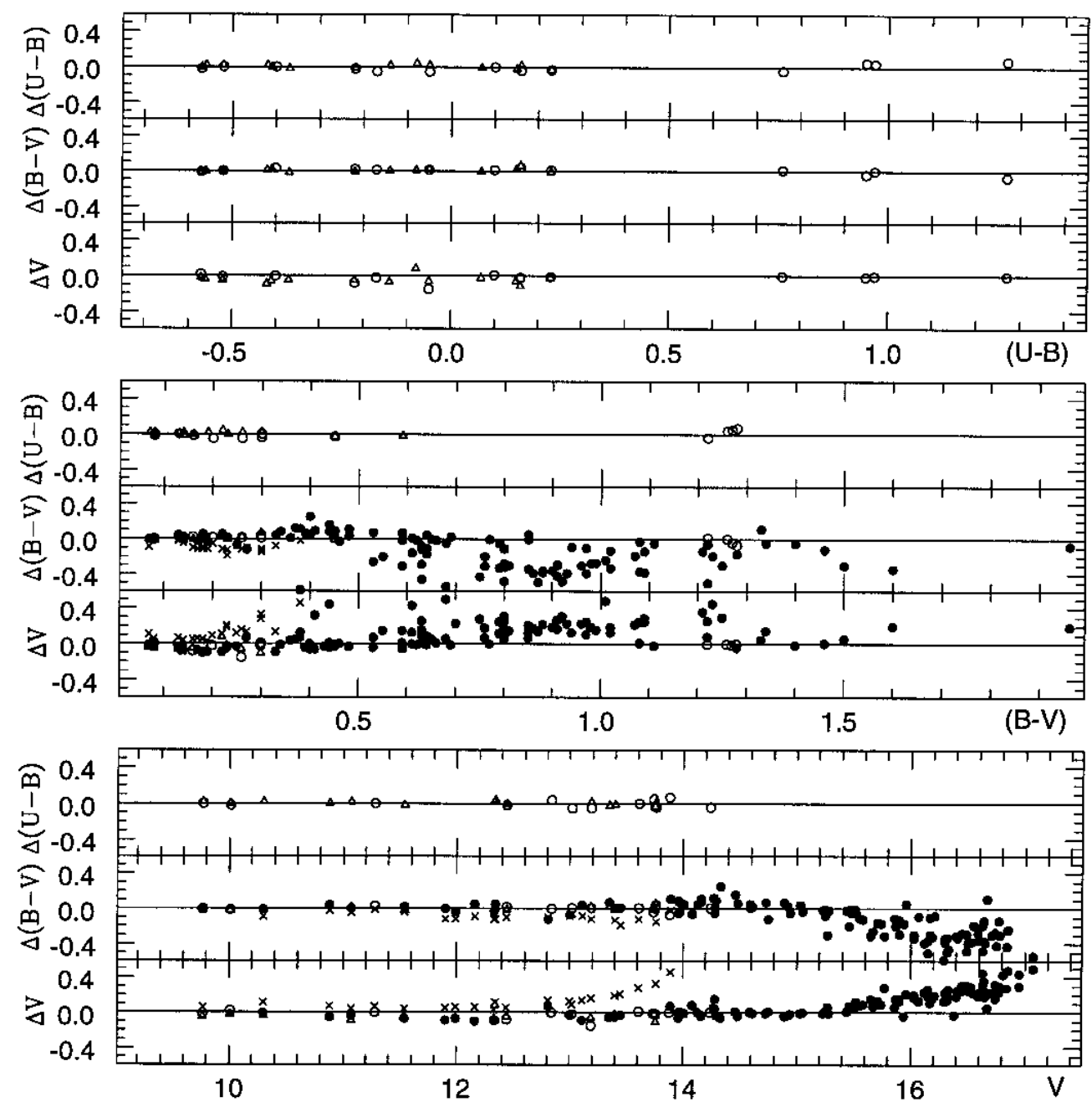

Fig. 5. A comparison of the present $U B V$ photometry in NGC 4103 with data given by Wesselink (1969), Becker et al. (1976) and Pedreros (1984). The differences $(\triangle)$ are in the sense present minus others' data, plotted against the present CCD photometry. Photoelectric data by Wesselink (1969) and Pedreros (1984) are denoted by open circles and triangles respectively, while photographic data by Wesselink (1969) and Becker et al. (1976) are denoted by filled circles and crosses respectively

Table 11. A comparison of the colour excess ratios with $E(B-V)$ for star clusters with the corresponding values for the normal interstellar extinction law given by Schmidt-Kaler (1982) for $E(U-B) / E(B-V)$; by Alcalá \& Ferro (1988) for $E(V-R) / E(B-V)$ and by Dean et al. (1978) for $E(V-I) / E(B-V)$

Object $\quad E(U-B) / E(B-V) \quad E(V-R) / E(B-V) \quad E(V-I) / E(B-V)$

Normal interstellar

NGC 4103

NGC 5662

0.72

NGC 6087

$0.67 \pm 0.02$

$$
\begin{gathered}
0.65 \\
0.60 \pm 0.06 \\
0.57 \pm 0.07 \\
0.54 \pm 0.10
\end{gathered}
$$

1.25

$1.41 \pm 0.09$

$1.41 \pm 0.20$

$1.17 \pm 0.10$ 

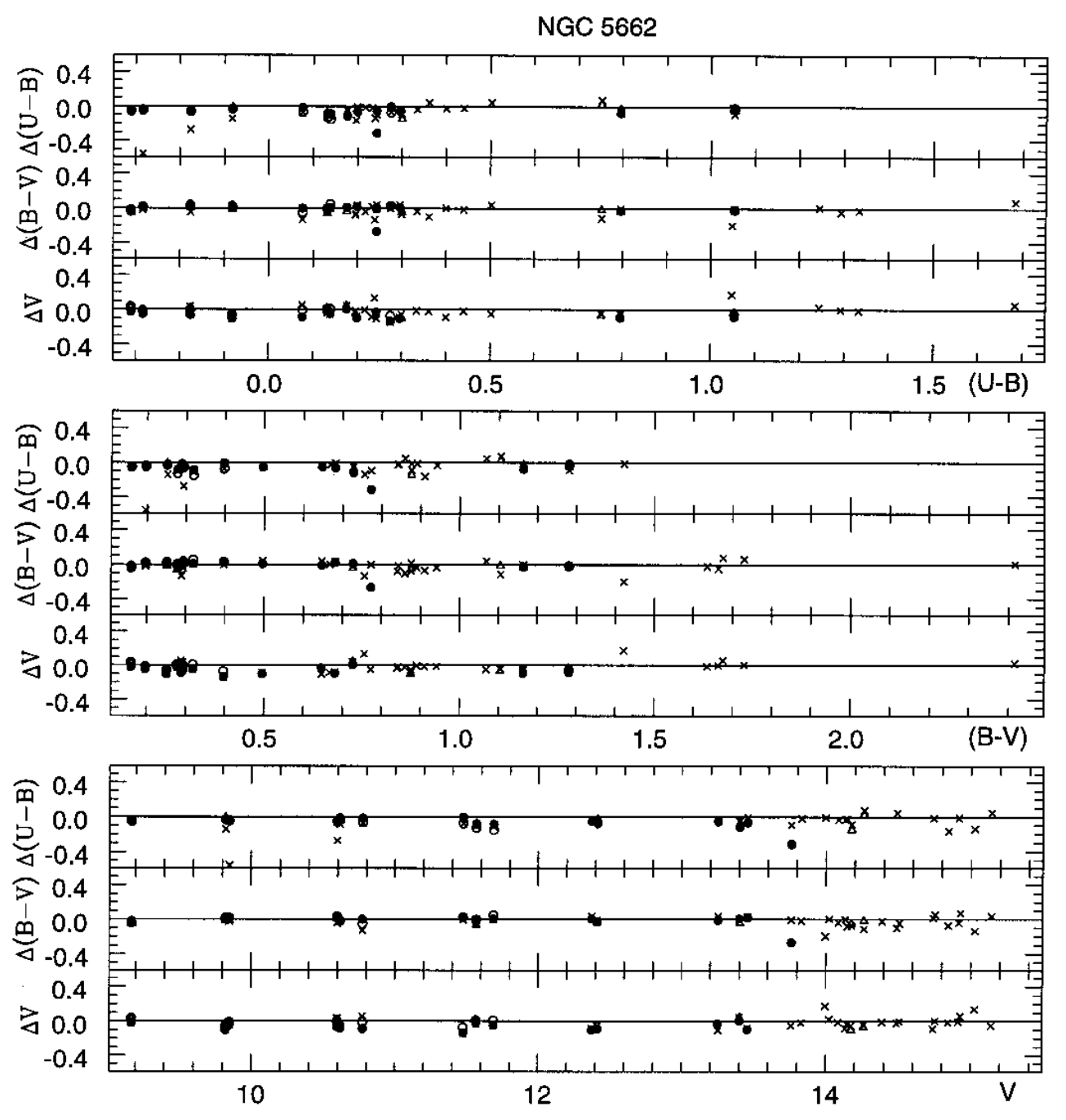

Fig. 6. A comparison of the present $U B V$ photometry in NGC 5662 with data given by Moffat \& Vogt (1973), Haug (1978) and Clariá et al. (1991). Other details are the same as in Figure 5. Photoelectric data by Moffat \& Vogt (1969), Haug (1978) and Clariá et al. (1991) are denoted by open circles, triangles and filled circles respectively, while photographic data by Haug (1978) are denoted by crosses

and

$$
\begin{aligned}
E(V-I)= & 1.25\left[1+0.06(B-V)_{0}\right. \\
& +0.014 E(B-V)] E(B-V)
\end{aligned}
$$

As the interstellar extinction seems to be uniform in front of the imaged cluster regions (see Sect. 6), we have used the same value of $E(B-V)$ for all the stars of a cluster.

In the $V_{0},(U-V)_{0}$ and $V_{0},(B-V)_{0}$ diagrams, we have fitted the ZAMS given by Schmidt-Kaler (1982), while the ZAMS given by Walker (1985) has been fitted in the $V_{0}$, $(V-R)_{0}$ and $V_{0},(V-I)_{0}$ diagrams. The $(V-R)_{0}$ colour for the ZAMS on the present photometric system was taken from Sagar \& Cannon (1994). After accounting for the colour dispersion expected from the error in observations, the visual fit of the ZAMS to the bluest envelope of the stars in each of the CM diagrams gives the $(m-M)_{0}$ values indicated in Figs. 13-16. The visual fit has been done mainly for stars brighter than $V_{0} \sim 14$ mag. Most of the stars in our sample of NGC 3228 are well below the cluster MS sequence defined by the brighter members having $U B V$ photoelectric data. Consequently, the $V_{0},(V-R)_{0}$ and $V_{0},(V-I)_{0}$ diagrams could not be used for the distance determination of this cluster.

The mean values of $(m-M)_{0}$ are $8.5 \pm 0.2,11.5 \pm 0.3$, $9.5 \pm 0.3$ and $9.9 \pm 0.3 \mathrm{mag}$ for NGC 3228, NGC 4103, NGC 5662 and NGC 6087 respectively. The uncertainty is estimated from the errors in $R, E(B-V)$ and the errors in fitting the ZAMS. The distance modulus determined above yields a distance of $500 \pm 50$ pc to NGC 3228 ; $2.0 \pm 0.3 \mathrm{kpc}$ to NGC $4103 ; 800 \pm 100$ pc to NGC 5662 and $960 \pm 130 \mathrm{pc}$ to NGC 6087. For NGC 4103, our value of distance modulus is in good agreement with the value of 11.35 given by Stetson (1981) and Becker (1971), but 
NGC 6087

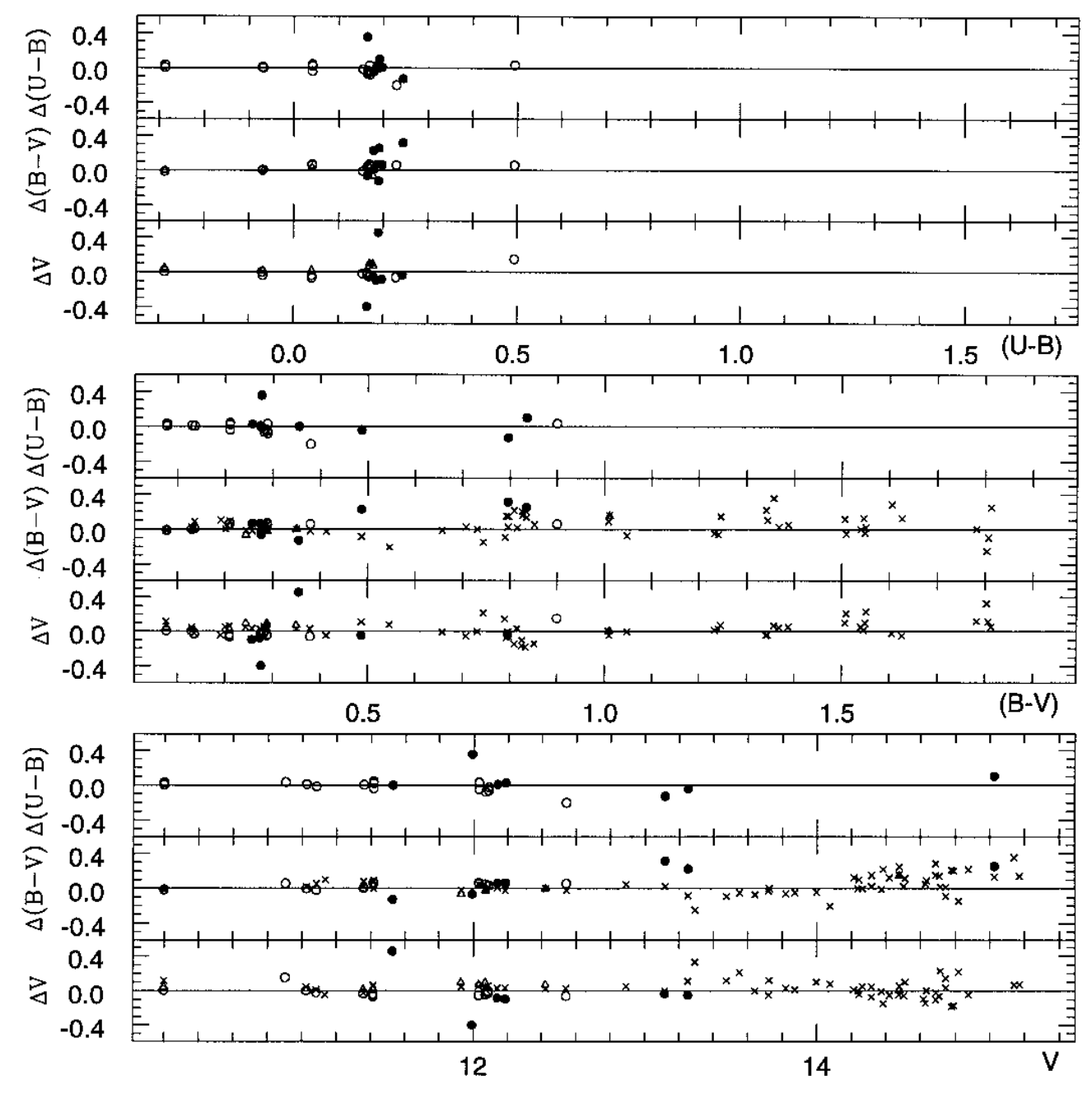

Fig. 7. A comparison of the present $U B V$ photometry in NGC 6087 with data given by Fernie (1961), Landolt (1964), Breger (1966), Pedreros (1984) and Turner (1986). Other details are the same as in Fig. 5. Photoelectric data by Fernie (1961) and Landolt (1964) are denoted by triangles, while those by Breger (1966), Pedreros (1984) and Turner (1986) are denoted by open circles. Photographic data by Landolt (1964) and Pedreros (1984) are denoted by crosses and filled circles respectively

somewhat larger than the value of 11.0 given by Wesselink (1969). The present determination of distance modulus for NGC 5662 is the same as that recently determined by Clariá et al. (1991). However, it is larger than the values of 8.82, 8.98 and 9.10 determined by Moffat \& Vogt (1973), Haug (1978) and Turner (1982) respectively. For NGC 6087, the values of distance modulus determined by Landolt (1964), Breger (1966) and Graham (1967) are 9.8, 9.7 and 9.9. They are in good agreement with the distance modulus determined by us. However, these values are larger than the values 9.4 and 9.6 given by Fernie (1961) and Schmidt (1980). The present determinations of the distances to the clusters should be reliable because they have been derived by fitting the ZAMS over a wide range of the unevolved part of the cluster MS, except in the case of NGC 3228.

\section{The CM diagrams of the clusters}

From the CM diagrams in Figs. 13-16, the following inferences can be drawn:

(i) The photoelectric data merge smoothly with the CCD observations, indicating that there are no systematic differences in the calibrations.

(ii) In all the star clusters under discussion, an evolutionary effect is clearly visible in the upper part of the cluster MS. In NGC 5662 and NGC 6087, a few proper motion cluster members have reached the giant phase of stellar evolution.

(iii) Well defined cluster main-sequences extending from $M_{v}=-4.5$ to +3.0 in NGC 4103 ; from $M_{v}=-2$ to +4 in NGC 5662 and from $M_{v}=-3$ to +4 in NGC 6087 are clearly seen. Such a long sequence is not present in 


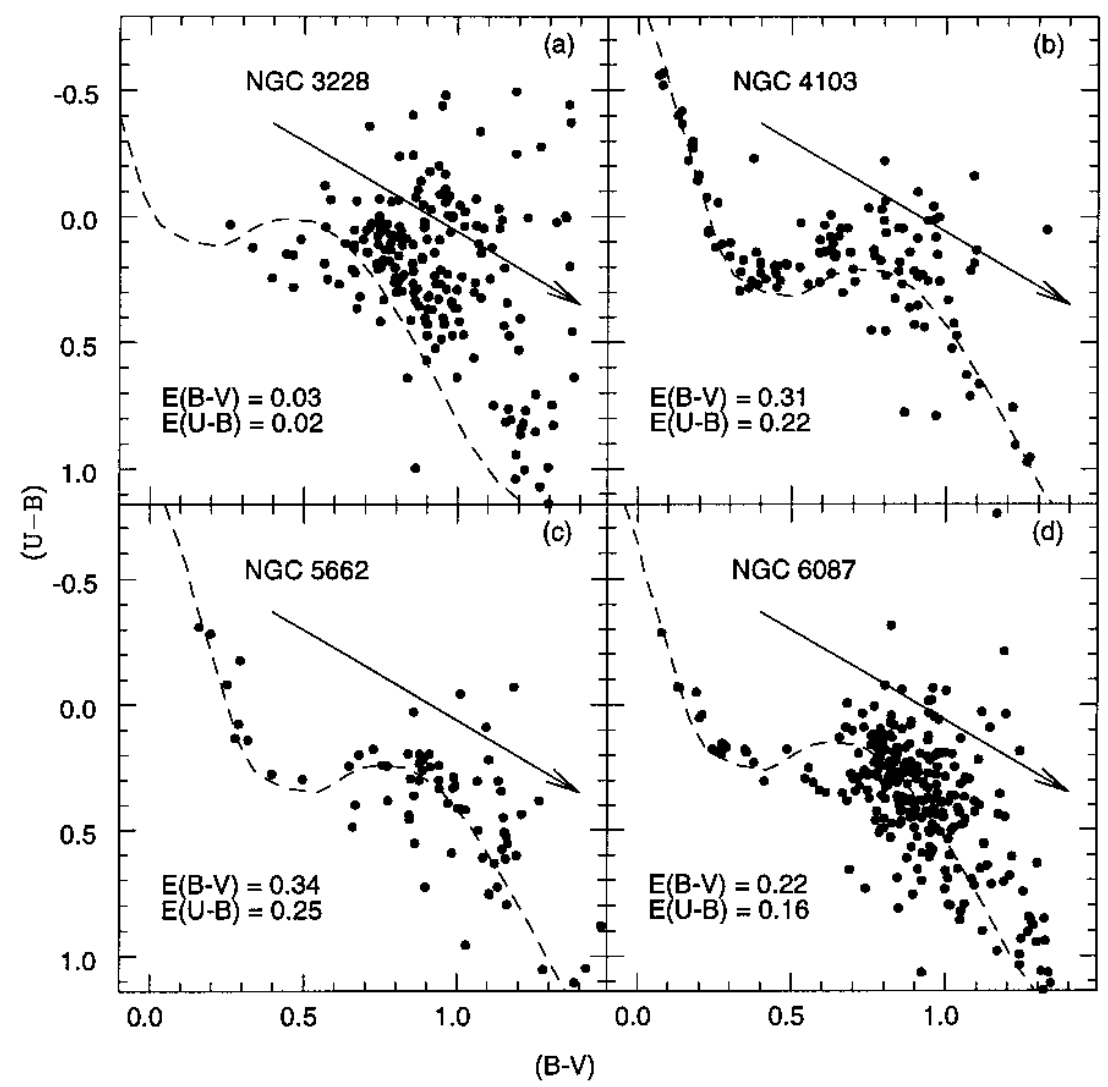

Fig. 8. The $(U-B)$ versus $(B-V)$ diagrams for the stars observed by us for NGC 3228 (panel a)), NGC 4103 b), NGC 5662 c) and NGC $6087 \mathbf{d})$. The continuous straight line represents the slope $(0.72)$ and direction of the reddening vector. The dotted curve represents the locus of Schmidt-Kaler's (1982) ZAMS, shifted in the direction of reddening by the values of $E(B-V)$ and $E(U-B)$ indicated in the diagram

NGC 3228 (Fig. 13). In fact very few cluster members seems to exist in the present sample of CCD data. It could be due to the fact that imaged regions are located almost at the edge of the cluster diameter (see Table 4).

(iv) It is clear that below $M_{v} \sim 0$, the luminosity functions of the clusters do not rise steeply enough to dominate the field star contamination. In this region, the present observations are unable to separate unambiguously cluster members from the field stars.

\section{Cluster membership of the Cepheid variables}

The positions of the Cepheid variables V Cen and S Norm are marked in the $V_{0},(U-V)_{0}$ and $V_{0},(B-V)_{0}$ diagrams of NGC 5662 (Fig. 15) and NGC 6087 (Fig. 16) respectively. For these, we used the $U B V$ data listed by Schaltenbrand \& Tammann (1971). The extremes of variability are also indicated.

Studies by Turner (1982) and more recently by Clariá et al. (1991) indicate that the Cepheid variable V Cen located in the coronal region of NGC 5662 is a cluster member. Similarly, studies by Feast (1957) and more re- cently by Turner (1986) and Mermilliod et al. (1987) confirm that the Cepheid variable $\mathrm{S}$ Norm, located near the optical centre of NGC 6087 , is a cluster member. As the present determination of distances to these clusters is accurate (since it is based on the CCD photometry of the unevolved part of the cluster MS), it may be worth while to discuss cluster membership of these variables in the light of our new data. Our distance determinations for NGC 5662 and NGC 6087 provide the values of $M_{V}=-3.5$ and -4.1 for $\mathrm{V}$ Cen and $\mathrm{S}$ Norm respectively. These luminosities agree very well with the corresponding values of -3.4 and -4.1 predicted from the period-luminosity relation of classical Cepheids given by Madore \& Freedman (1991). The location of these Cepheids in the CM diagrams fits very well with the stellar evolutionary isochrone appropriate for the average age of their parent star clusters (see Figs. 15 and 16). The values of $E(B-V)$ derived by Dean et al. (1978) are 0.29 and $0.18 \mathrm{mag}$ for V Cen and $\mathrm{S}$ Norm respectively. These values agree fairly well with the corresponding values of $E(B-V)$ derived by us for NGC 5662 and NGC 6087 (see Sect. 6). All these various pieces of evidence strongly suggest that the 


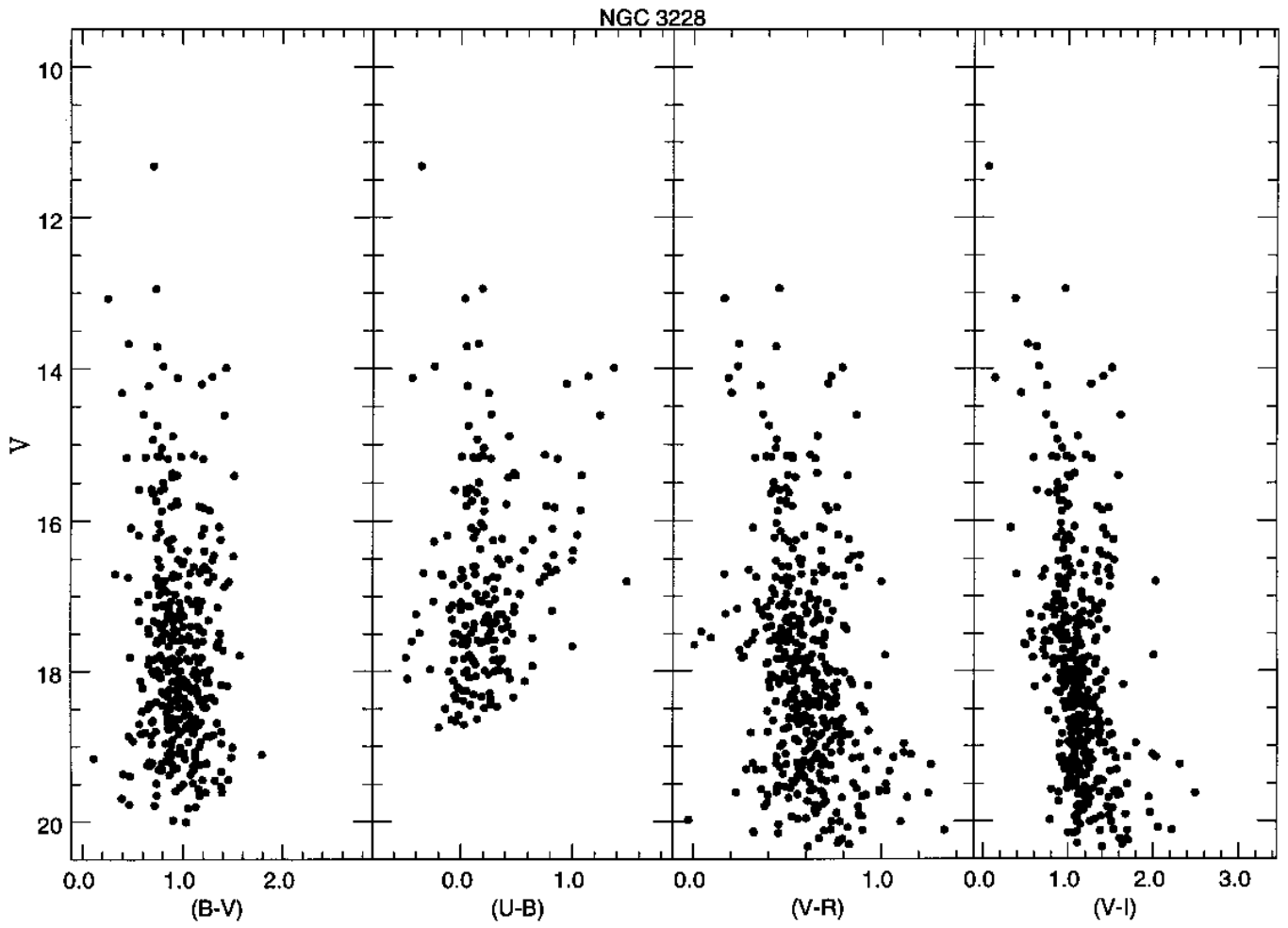

Fig. 9. The $V,(B-V) ; V,(U-B) ; V,(V-R)$ and $V,(V-I)$ diagrams for the stars observed by us in NGC 3228

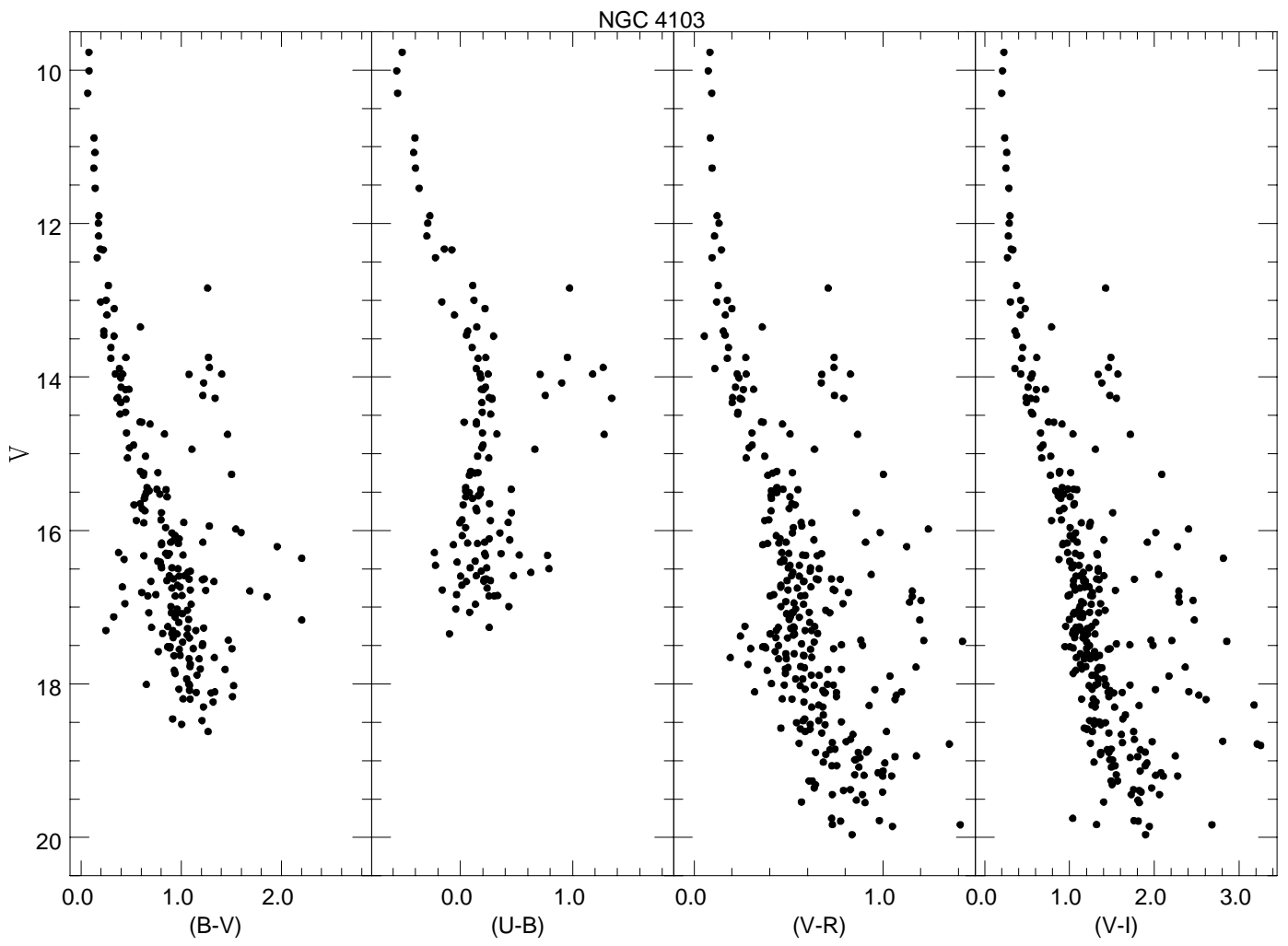

Fig. 10. The $V,(B-V) ; V,(U-B) ; V,(V-R)$ and $V,(V-I)$ diagrams for the stars observed by us in NGC 4103 


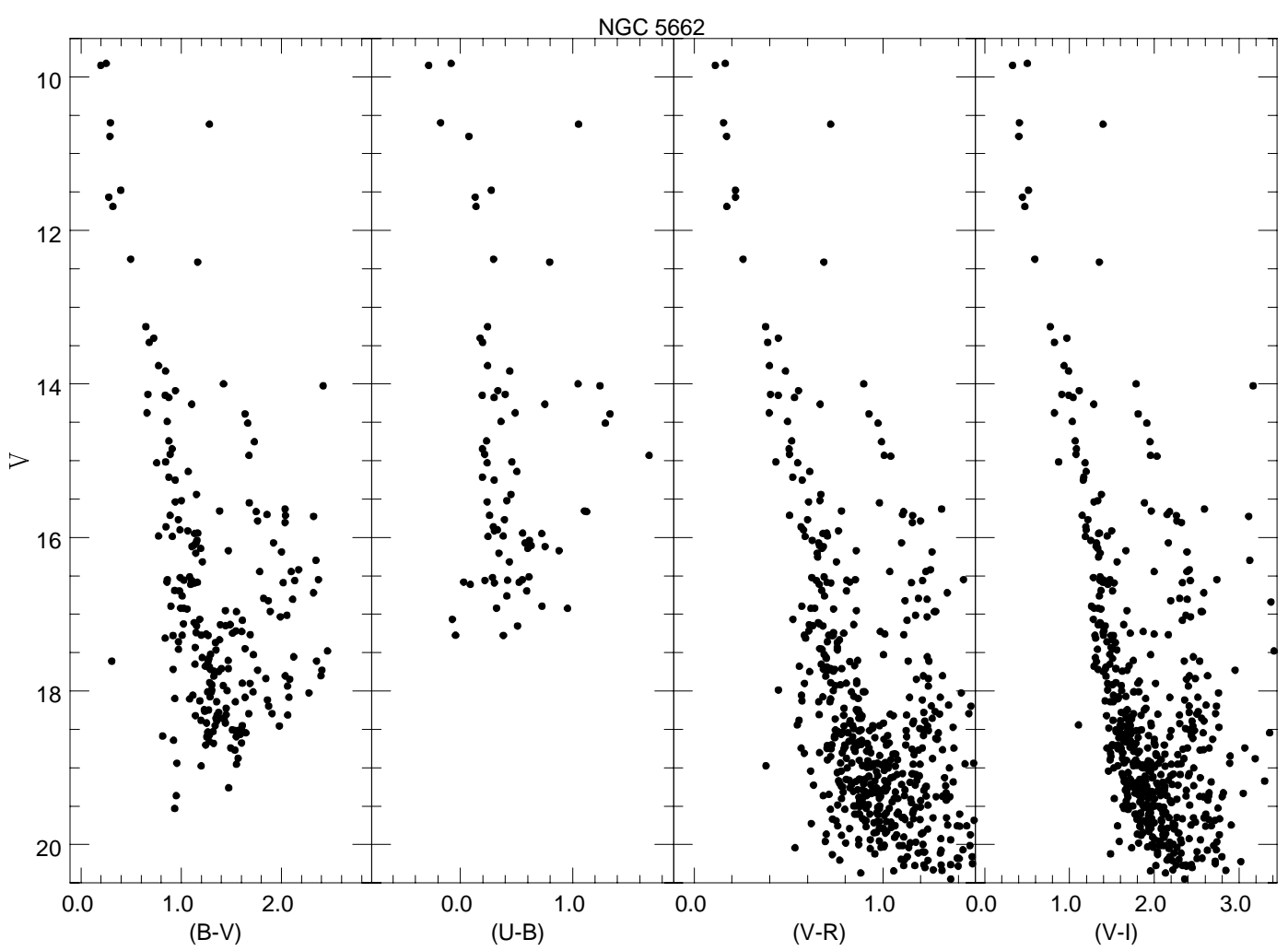

Fig. 11. The $V,(B-V) ; V,(U-B) ; V,(V-R)$ and $V,(V-I)$ diagrams for the stars observed by us in NGC 5662

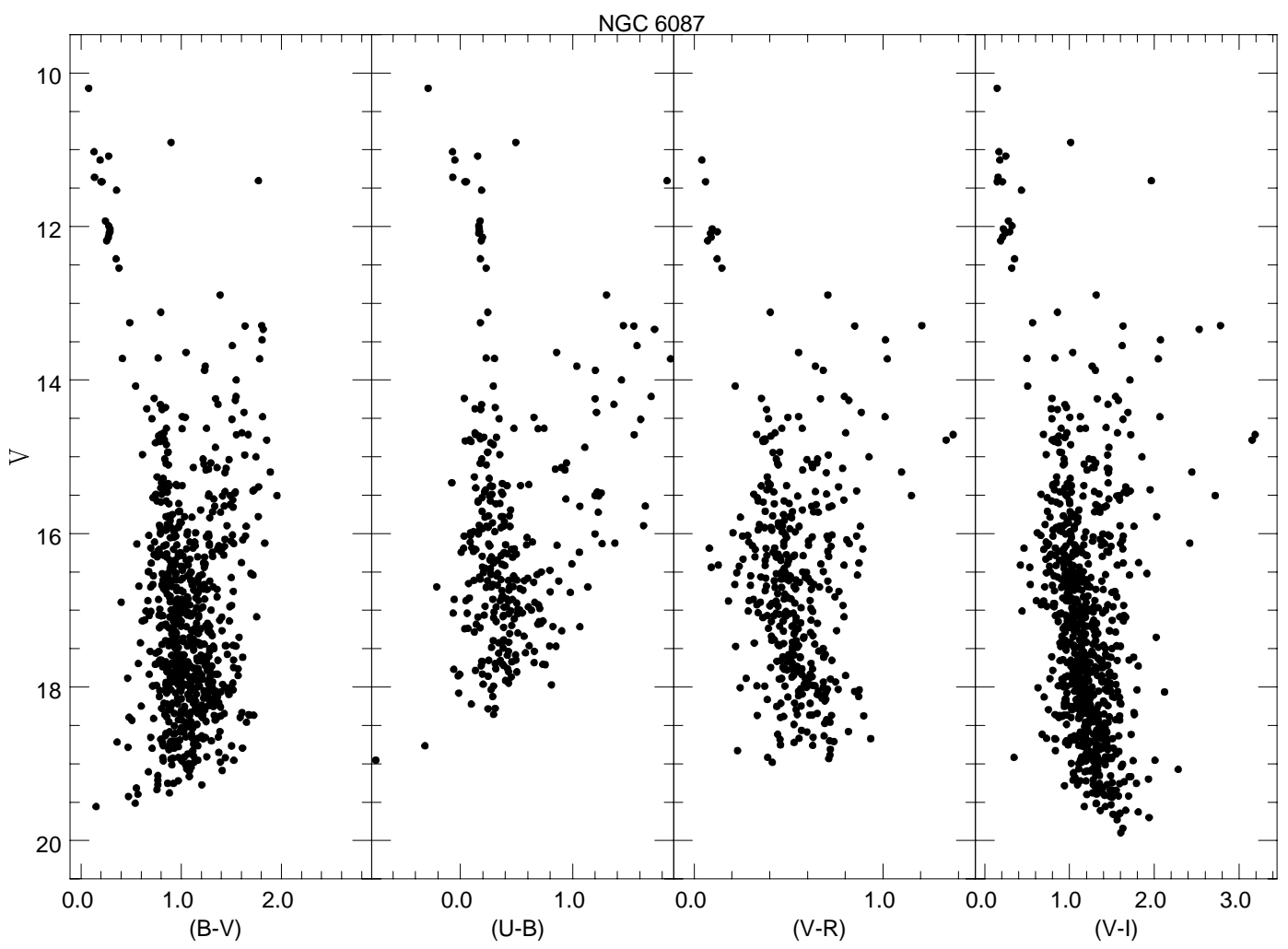

Fig. 12. The $V,(B-V) ; V,(U-B) ; V,(V-R)$ and $V,(V-I)$ diagrams for the stars observed by us in NGC 6087 


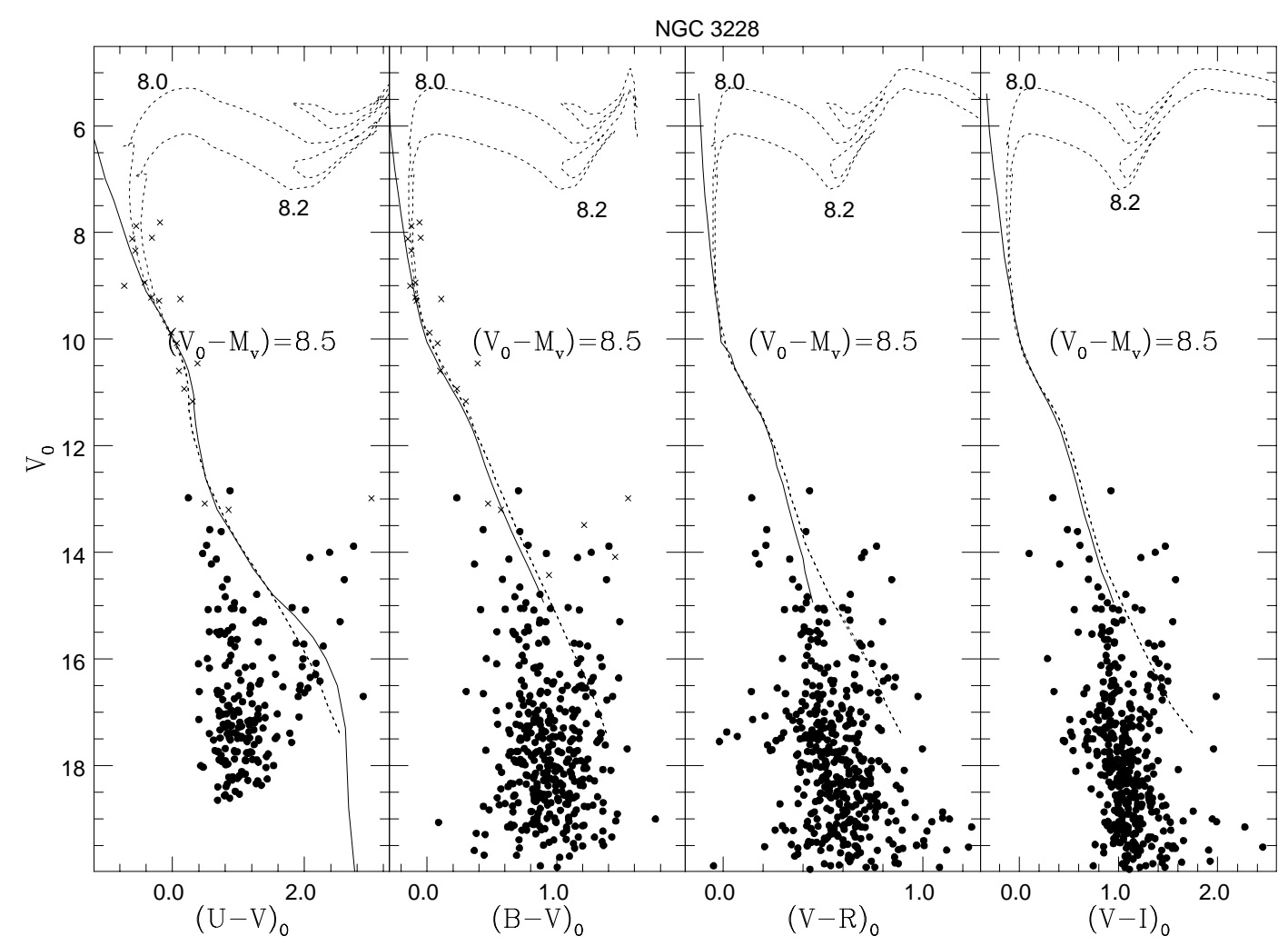

Fig. 13. The $V_{0},(U-V)_{0} ; V_{0},(B-V)_{0} ; V_{0},(V-R)_{0}$ and $V_{0},(V-I)_{0}$ diagrams for stars observed by us (filled circles), as well as for stars (crosses) observed photoelectrically in the $U B V$ passbands by Hogg (1963), in NGC 3228. The continuous curves are the ZAMS fitted to the unevolved part of the cluster MS for the values indicated in the diagram. The dotted curves are the isochrones for Pop I stars fitted to the bright cluster members for the values (in log of years) indicated in the diagram. The mean value of the distance modulus $(m-M)_{0}$ to the cluster is $8.5 \mathrm{mag}$, while its age is $\sim 100 \mathrm{Myr}$

Cepheid variables V Cen and S Norm are indeed member of the open star clusters NGC 5662 and NGC 6087 respectively.

\section{Ages of the cluster members}

The brighter stars in each cluster under discussion show evolutionary effects. Most of them are confirmed proper motion cluster members. The ages of these stars have been determined by fitting the theoretical stellar evolutionary isochrones given by Bertelli et al. (1994) in the corresponding CM diagrams (Figs. 13-16). The isochrones are for Pop I stars $(X=0.7, Y=0.28$ and $Z=0.02)$ and include the effects of convective core overshooting in the models. The isochrone fitting to the evolved MS, and to the few bright red giants in NGC 5662 and NGC 6087, indicates that bright stars have mean ages of $100 \mathrm{Myr}$ in NGC 3228; of $30 \mathrm{Myr}$ in NGC 4103; of $80 \mathrm{Myr}$ in NGC 5662 and of $65 \mathrm{Myr}$ in NGC 6087. In the case of NGC 3228 , the age is estimated only from the $V_{0},(U-V)_{0}$ and $V_{0},(B-V)_{0}$ diagrams (Fig. 13); as the brighter evolved cluster members do not have $R$ and $I$ magnitudes.

The present age estimate for NGC 3228 is lower than the 250 Myr age estimated by Hogg (1963) but in good agreement with the value of 130 Myr given in Lynga's (1987) catalogue. For NGC 4103, our age estimate is almost the same as given by Wesselink (1969). However, it is slightly lower than the value of 40 Myr given in Lyngå's (1987) catalogue. The age of NGC 5662 derived by us is in good agreement with the values of 60, 80 and $79 \mathrm{Myr}$ given by Haug (1978), Lyngå (1987) and Clariá et al. (1991) respectively. Similarly, our age estimate for NGC 6087 is in good agreement with the values of 50 and $70 \mathrm{Myr}$ determined by Turner (1986) and Lyngå (1987) respectively.

\section{Field star contamination in the clusters}

Stars below $M_{v}=+5.0$ in NGC 3228; below $M_{v}=-1.0$ in NGC 4103; below $M_{v}=-3.0$ in NGC 5662 and below $M_{v}=0.5$ in NGC 6087 show a large spread in colour at a given brightness level, compared to that expected from observational errors and differential reddening. The main reasons for such a spread could be the presence of field stars; binaries and peculiar cluster members. The field stars located in the galactic plane, at about the cluster distances and with similar reddenings, will occupy almost the same area in the colour-colour (CC) and CM diagrams as the cluster members. It is therefore difficult to separate 


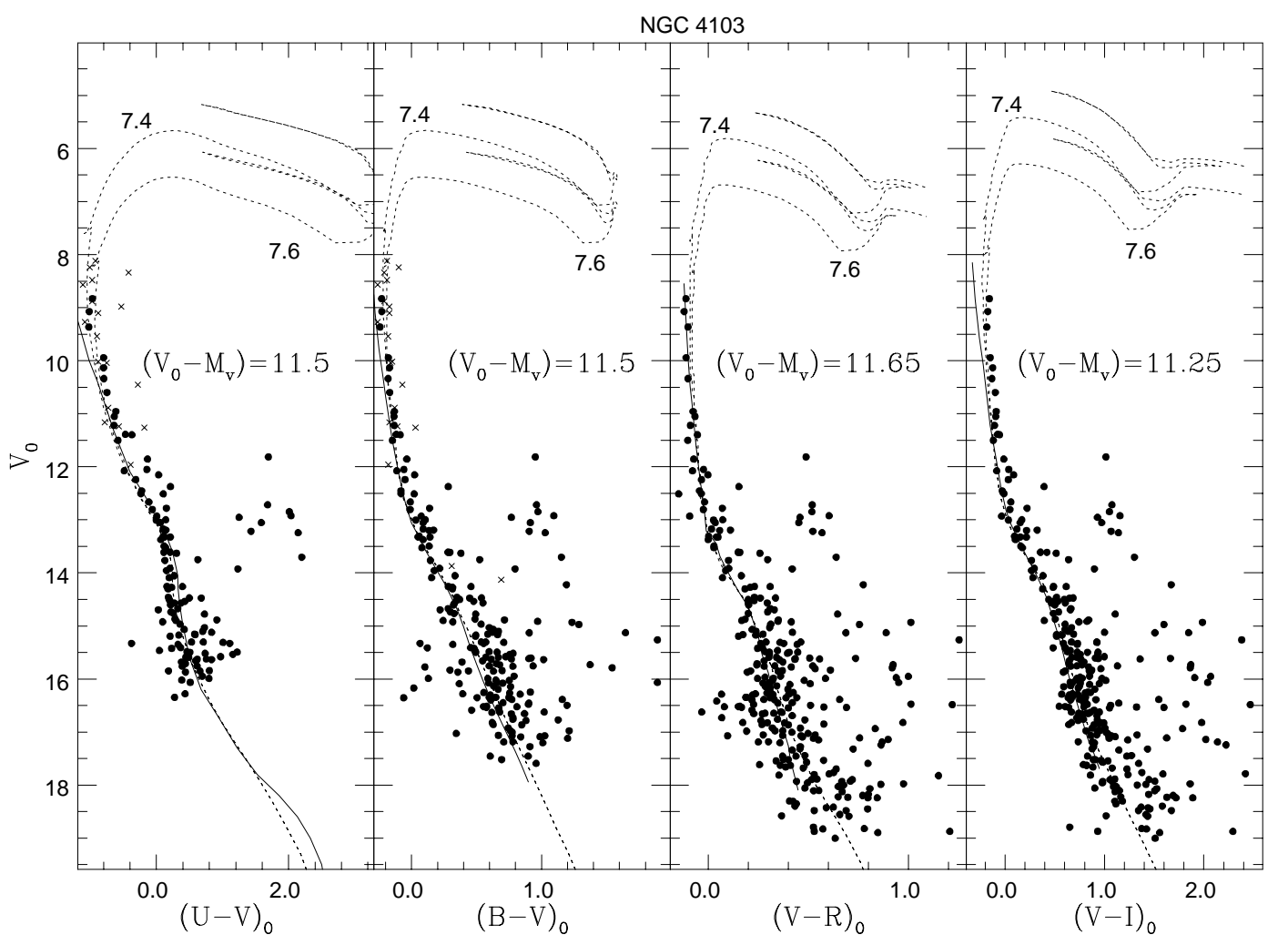

Fig. 14. The $V_{0},(U-V)_{0} ; V_{0},(B-V)_{0} ; V_{0},(V-R)_{0}$ and $V_{0},(V-I)_{0}$ diagrams for stars observed by us (filled circles), as well as for stars (crosses) observed photoelectrically in the $U B V$ passbands by Wesselink (1969), in NGC 4103. Others lines are the same as in Fig. 13. The mean value of the distance modulus $(m-M)_{0}$ to $\sim 35$ Myr old cluster is 11.5 mag

cluster members from field stars only on the basis of their closeness to the main populated area of the $\mathrm{CM}$ and $\mathrm{CC}$ diagrams. However, the possibility of cluster membership is small for stars located well away from the cluster MS in most of the CM and CC diagrams. As the present observations are unable to quantify the field star populations in the vicinity of clusters under study, the statistically expected number of cluster members among the fainter stars can not be determined in the same way as we did in NGC 4755 (Sagar \& Cannon 1995). However, the following procedure has been adopted for this purpose. The frequency distributions of stars are derived from the $(V-I)_{0} \mathrm{CM}$ diagrams (see Table 12). These diagrams were preferred over other $\mathrm{CM}$ diagrams because of better data completeness in them. Inspection of our CCD frames and an estimate of the numbers of crowded images indicate that we are $\sim 85 \%$ complete in general. To avoid the effect of relatively large $(\geq 50 \%)$ data incompleteness, the analysis was restricted to the brightness level which is about 1 mag above the limiting magnitude of our observations. To derive the frequency distribution of stars, the $(V-I)_{0} \mathrm{CM}$ diagram is divided into two magnitude bins, namely brighter and fainter; and into three colour bins called "below", "near" and "above MS". The brighter magnitude bin represents the region in which the cluster main sequences are clearly visible, while in the fainter magnitude bin, any cluster sequence is hardly distinguishable from the field star contamination. Stars lying within two standard deviations in $(V-I)_{0}$ either side of the ZAMS are put in the colour bin called "near MS" and $N_{\mathrm{MS}}$ denotes number of such stars in Table 12. The width of the colour bins increases with decreasing brightness. Stars located towards the blue and red sides of this bin are put in the colour bins named "below MS" and "above MS" respectively. $N_{\text {BMS }}$ and $N_{\text {AMS }}$ denote the corresponding numbers in Table 12. This table clearly shows that:

(i) in the brighter magnitude bin for a cluster, the number of stars in the "near MS" bin are significantly larger than either $N_{\mathrm{BMS}}$ or $N_{\mathrm{AMS}}$, indicating that the cluster sequences are generally well defined (see Sect. 8).

(ii) in the fainter magnitude bin for NGC 3228 and NGC 6087, the numbers of stars below the MS are very large in comparison to the value of $N_{\mathrm{MS}}$. This may indicate that background field star contamination is very large in the direction of the imaged regions of these clusters. In the case of NGC 4103 and NGC 5662, foreground field star contamination seems to be more dominant. In order to get an idea of the number of cluster members which might be present in $N_{\mathrm{MS}}$ for the fainter bin, we performed the following simple exercise. 


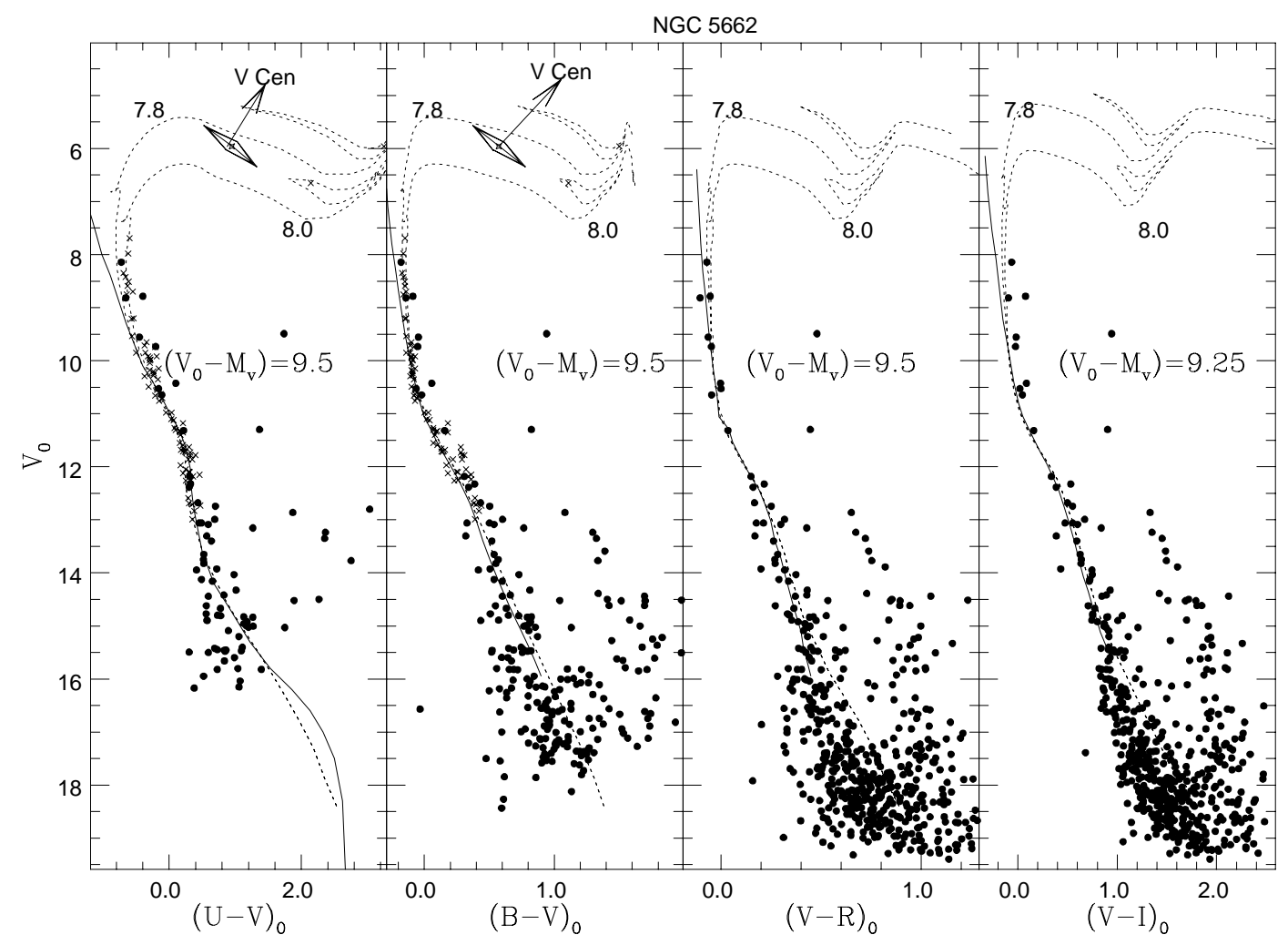

Fig. 15. The $V_{0},(U-V)_{0} ; V_{0},(B-V)_{0} ; V_{0},(V-R)_{0}$ and $V_{0},(V-I)_{0}$ diagrams for stars observed by us (filled circles), as well as for stars (crosses) observed photoelectrically in the $U B V$ passbands by Clariá (1991), in NGC 5662. Other details are the same as in Fig. 13. The mean value of the distance modulus $(m-M)_{0}$ to the cluster is 9.5 mag, while its age is $\sim 80 \mathrm{Myr}$

Table 12. Frequency distribution of the stars in the $M_{v}$, $(V-I)_{0}$ diagram for the clusters under study derived from our sample. $N_{\mathrm{BMS}}, N_{\mathrm{MS}}$ and $N_{\mathrm{AMS}}$ denote number of stars below, near and above the ZAMS respectively while $N_{\mathrm{EX}}$ denotes number of cluster members expected in the fainter magnitude bin assuming Salpeter mass function slope

$\begin{array}{clllll}\text { Cluster } & \begin{array}{l}M_{v} \text { range } \\ \text { (mag) }\end{array} & N_{\mathrm{BMS}} & N_{\mathrm{MS}} & N_{\mathrm{AMS}} & N_{\mathrm{EX}} \\ & & & & & \\ \text { NGC 3228 } & +4.0 \text { to }+6.5 & 2 & 13 & 4 & \\ & +6.5 \text { to }+11.8 & 317 & 43 & 9 & 75 \\ \text { NGC 4103 } & -3.0 \text { to }+2.0 & 0 & 35 & 10 & \\ & +2.0 \text { to }+6.5 & 6 & 138 & 81 & 106 \\ \text { NGC 5662 } & -1.5 \text { to }+4.0 & 0 & 20 & 6 & \\ & +4.0 \text { to }+9.0 & 31 & 227 & 146 & 58 \\ & & & & & \\ \text { NGC } 6087 & -0.5 \text { to }+4.5 & 0 & 46 & 34 & \\ & +4.5 \text { to }+8.5 & 387 & 266 & 41 & 84\end{array}$

Assuming that (a) the mass function of the clusters under discussion can be represented by Salpeter's (1955) mass function (which may be justified in the light of recent studies of young star clusters of our Galaxy (cf. Sagar et al. 1986; Sagar 1993; Phels \& Janes 1993) as well as of the Magellanic Clouds (cf. Sagar \& Richtler 1991; Sagar 1993 and references therein)) and (b) all the stars near the MS in the brighter bin are cluster members, the number of cluster members expected $\left(N_{\mathrm{EX}}\right)$ in the fainter bin are derived. They are listed in the last column of the Table 12. The luminosity functions of the $N_{\mathrm{MS}}$ and $N_{\mathrm{EX}}$ are presented in Fig. 17 by solid and dotted histograms respectively. Except for NGC 3228 , the value of $N_{\mathrm{EX}}$ is always less than $N_{\mathrm{MS}}$ indicating clearly that not all MS stars are cluster members. A comparison of these numbers and Fig. 17 indicate that large field star contamination is present in the case of NGC 5662 and NGC 6087. To know the actual number of cluster members in the $N_{\mathrm{MS}}$ of the fainter bin, their precise proper motions and/or radial velocities are required. In the absence of such data for the stars observed by us, it is difficult to separate unambiguously the cluster members from the field stars. Nevertheless, there is evidence from visual inspection of several of the cluster CM diagrams that the number of intermediate brightness stars does not increase as rapidly as one might expect, down to the level at which field star contamination makes it impossible to recognise the cluster main sequence. This is 


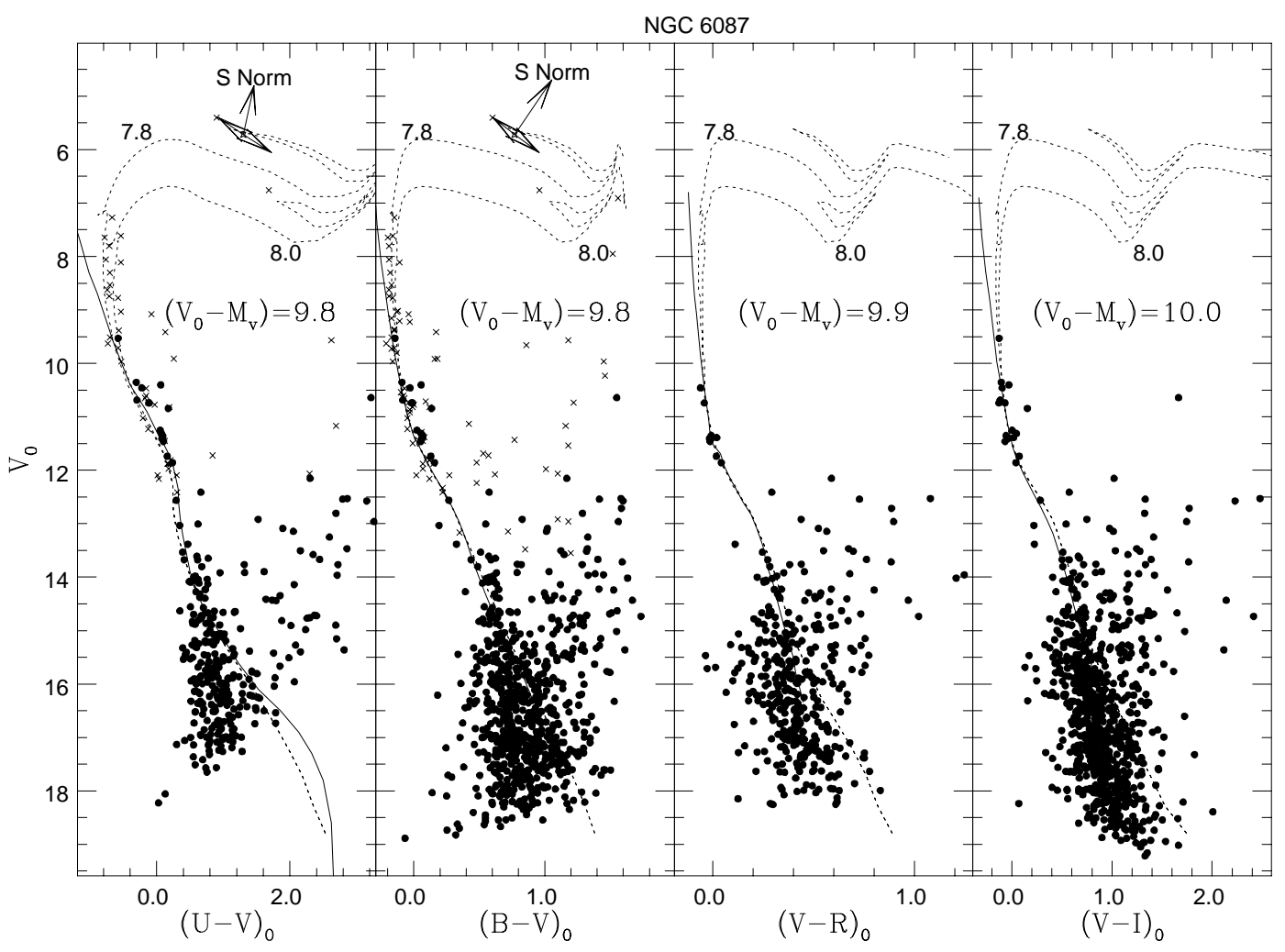

Fig. 16. The $V_{0},(U-V)_{0} ; V_{0},(B-V)_{0} ; V_{0},(V-R)_{0}$ and $V_{0},(V-I)_{0}$ diagrams for stars observed by us (filled circles), as well as for stars (crosses) observed photoelectrically in $U B V$ passbands by Fernie (1961), Breger (1966) and Turner (1986), in NGC 6087. Other details are the same as in Fig. 13. The mean value of the distance modulus $(m-M)_{0}$ to the cluster is 9.9 mag. The object is $\sim 65 \mathrm{Myr}$ old

particularly true in the case of NGC 6087 , where there seems to be a real deficiency of stars for $+2<M_{v}<$ +4 . The small proportion of faint cluster members, and the low gradient of cluster number density across our relatively small fields, clearly make it impossible to use the spatial distribution to help select cluster members or locate the faint MS.

\section{Possible pre-MS cluster members}

To see whether the present observations have reached to the faintness level of PMS stars or not, the $M_{v}$, $(B-V)_{0}$ diagrams for the stars observed by us (filled circles), as well as for the members (generally proper motion) observed photoelectrically by others (crosses) but not present in our sample have been plotted in Fig. 18 for all the star clusters under study. In plotting this figure, we used uniform interstellar extinction with values of $E(B-V)=0.03,0.31,0.34$ and 0.22 for NGC 3228, NGC 4103, NGC 5662 and NGC 6087 respectively. The corresponding values used for their true distance moduli are 8.5, 11.5, 9.5 and 9.9. In this diagram, we have also plotted Iben's (1965) isochrones for contracting stars with ages 10 and $30 \mathrm{Myr}$ and the theoretical stellar birthline given by Palla \& Stahler (1992), which marks the lumi- nosity and temperature at which contracting stars are expected first to become visible at optical wavelengths, together with the ZAMS. The following inferences can be drawn from this figure:

(i) A few stars in NGC 4103 and NGC 6087 are located well above the theoretical stellar birthline. They are probably field stars.

(ii) No PMS is clearly visible in the clusters under study. The low-mass stars which formed at the same time as the more massive stars are expected to join the ZAMS at $M_{v}=+6.6,+4.1,+6.0$ and +5.8 for the clusters NGC 3228, NGC 4103, NGC 5662 and NGC 6087 respectively. So the present observations have barely reached to the faintness level for detecting the PMS stars. Also to make sure, one should look for signatures of PMS stars like $\mathrm{H}_{\alpha}$ emission, irregular variability or ultraviolet excess in them, as Adams et al. (1983) did in NGC 2264. On the other hand, our observations in NGC 4755 have been able to detect PMS stars (see Sagar \& Cannon 1995). NGC 4755 is the youngest ( $\sim 10 \mathrm{Myr})$ open star cluster observed under this programme. The clearly visible kink away from the ZAMS around $M_{v}=+3.5$ in $M_{v},(B-V)_{0}$ diagram of NGC 4755 (see Fig. 7 in Sagar \& Cannon 1995) is 


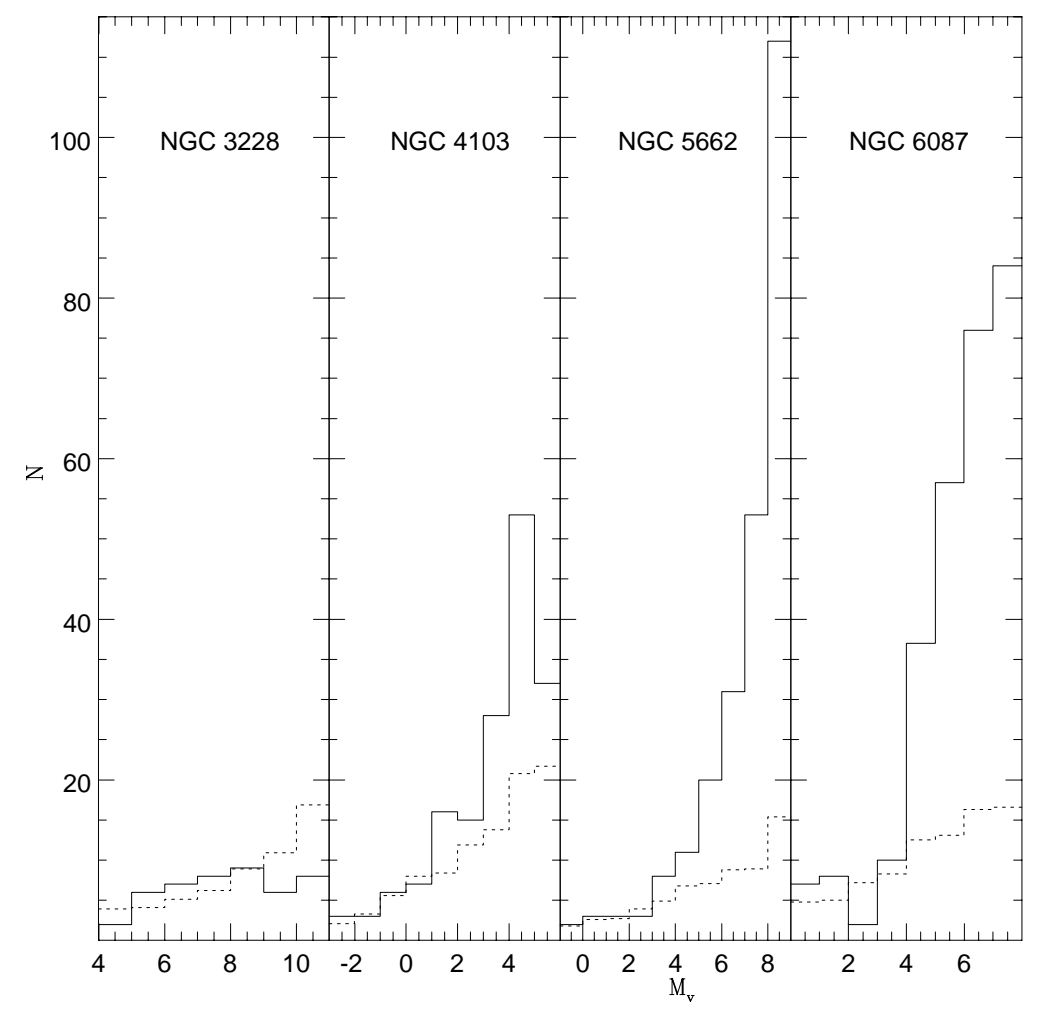

Fig. 17. The luminosity functions of the "near MS" $\left(N_{\mathrm{MS}}\right)$ stars and the expected cluster members $\left(N_{\mathrm{Ex}}\right)$ are shown by solid and dotted histograms respectively. $N$ is the number of stars in one magnitude bin of $M_{v}$
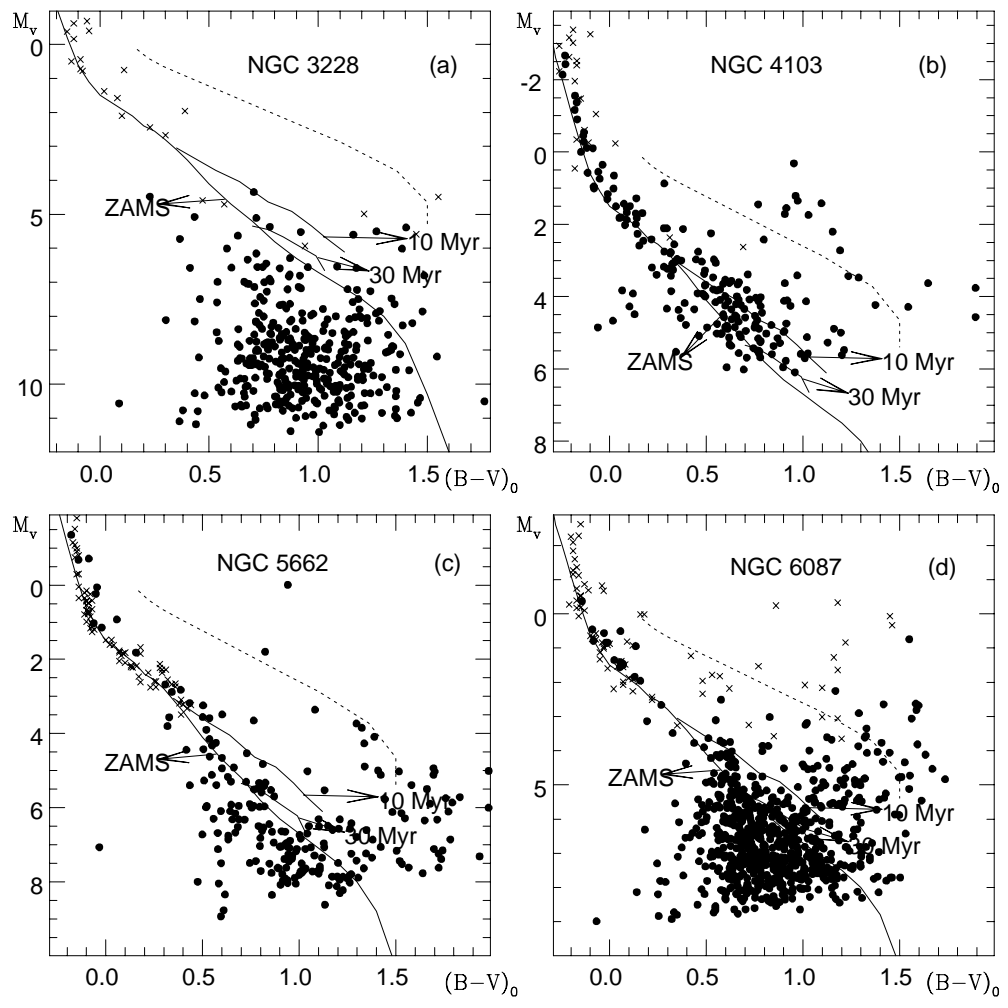

Fig. 18. The $M_{v},(B-V)_{0}$ diagrams are plotted again in a), b), c) and d) for NGC 3228 , NGC 4103 , NGC 5662 and NGC 6087 respectively. Theoretical stellar evolutionary isochrones taken from Iben (1965) for the pre-MS phase of evolution are plotted. The theoretical stellar birthlines taken from Palla \& Stahler (1992) are shown by dotted lines 
interpreted as the MS turn-on for PMS stars. Most of the PMS stars have an age around $6 \mathrm{Myr}$, indicating that massive stars in NGC 4755 formed about 4 Myr before the bulk of the low-mass stars.

\section{Conclusions}

New $U B V R I$ CCD photometry down to $V \sim 20$ is presented for about 2000 stars altogether near the young open clusters NGC 3228, NGC 4103, NGC 5662 and NGC 6087. The present work in combination with earlier proper motion, radial velocity, photoelectric and spectroscopic observations leads to the following conclusions:

(i) Visual fitting of the ZAMS to the bluest envelope of the cluster sequence in the $V_{0},(B-V)_{0} ; V_{0},(U-V)_{0}$; $V_{0},(V-R)_{0}$ and $V_{0},(V-I)_{0}$ diagrams over a broad range of $V(\sim 7 \mathrm{mag})$ gives distances of $2.0 \pm 0.3 \mathrm{kpc}$ to NGC 4103, $0.8 \pm 0.1 \mathrm{kpc}$ to NGC 5662 and $1.0 \pm 0.1 \mathrm{kpc}$ to NGC 6087 .

(ii) Reddening across the observed regions in NGC 4103, NGC 5662 and NGC 6087 seems to be uniform with mean values of $E(B-V)=0.31,0.34$ and 0.22 respectively. The law of interstellar extinction in the direction of these cluster regions is normal.

(iii) The present work supports earlier finding that the Cepheid variables V Cen and S Norm are probably members of NGC 5662 and NGC 6087 respectively.

(iv) Bertelli et al. (1994) isochrones fitted to the bright cluster members in the $V_{0},(U-V)_{0} ; V_{0},(B-V)_{0}$; $V_{0},(V-R)_{0}$ and $V_{0},(V-I)_{0}$ diagrams indicate that the oldest cluster in the sample is NGC 3228 with an age of $\sim 100$ Myr and the youngest is NGC 4103 with an age of $\sim 30$ Myr. Both NGC 5662 and NGC 6087 are of nearly the same age, having values $\sim 80$ and $65 \mathrm{Myr}$ respectively.

(v) It is difficult to separate cluster members from field stars on the basis of present observations alone; kinematic data are also required. Deep higher precision photometric and multi-object spectroscpic observations in the optical as well as in the near infrared are required to identify the PMS stars in the clusters under discussion.

Acknowledgements. We thank the Mount Stromlo and Siding Spring Observatories of the Australian National University for the allotment of observing time for this project and the referee Dr. J.J. Clariá for constructive comments. Financial support for RS provided by the then Department of Industry, Technology and Commerce of Australia, by the IAU and by the AAO are gratefully acknowledged.
This research has made use of the Open Cluster Data Base compiled by J.-C. Mermilliod, Institut d'Astronomie de l'Université de Lausanne, Switzerland.

\section{References}

Alcalá J.M., Ferro A.A., 1988, Rev. Mex. Astron. Astrofis 16, 81

Adams M.T., Strom K.M., Strom S.E., 1983, ApJS 53, 893

Becker W., Svolopoulos S.N., Fang Ch., 1976, Astron. Inst. Univ. Basel Kataloge photographischer und photoelektrischer helligkeiten von 25 galaktischen Sternhaufen im RGU- und $U B V$-system

Bell S.A, Kilkenny D., Malcolm G.J., 1987, MNRAS 226, 879

Breger M., 1966, PASP 78, 293

Bertelli G., Bressan A., Choisi C., Fagotto F., Nasi E., 1994, A\&AS 106, 275

Clariá J.J., Lapasset E., Bosio M.A., 1991, MNRAS 249, 193

Dean F.J., Warren P.R., Cousins A.W.J., 1978, MNRAS 183, 569

Eggen O.J., 1980, ApJ 238, 919

Feast M.W., 1957, MNRAS 117, 193

Fernie J.D., 1961, ApJ 133, 64

Fitzgerald M.P, Luiken M., Maitzen H.M., Moffat A.F.J., 1979, A\&AS 37, 345

Johnson H.L., Morgan W.W., 1953, ApJ 117, 313

Graham J.A., 1967, MNRAS 135, 377

Graham J.A., 1982, PASP 94, 244

Hartoog M.R., 1976, ApJ 205, 807

Hogg A.R., 1963, MNRAS 125, 307

Haug U., 1978, A\&AS 34, 417

Iben I. Jr., 1965, ApJ 141, 993

Irwin J.B., 1958, AJ 63, 46

Kamp L.W., 1974, A\&AS 16, 1

King D.S., 1979, J. Proc. R. Soc. N.S.W. 112, 13 (三 Sydney Obs. Papers No. 83)

King D.S., 1980, J. Proc. R. Soc. N.S.W. 113, 7 (三 Sydney Obs. Papers No. 87)

King D.S., 1982, J. Proc. R. Soc. N.S.W. 115, 1 (三 Sydney Obs. Papers No. 93)

Landolt A.U., 1964, ApJS 8, 329

Luck R.E., 1994, ApJS 91, 309

Lyngå G., 1987, Catalogue of Open Cluster Data, 5th edition, 1/1 S7041, Centre de Données Stellaires, Strasbourg

Madore B.F., Freedman W.L., 1991, PASP 103, 933

Mermilliod J.-C., 1992, Bull. Inf. CDS 40, 115

Mermilliod J.-C., Mayor M., Burki, G., 1987, A\&AS 70, 389

Moffat A.F.J., Vogt N., 1973, A\&AS 10, 135

Palla F., Stahler S.W., 1992, ApJ 392, 667

Pedreros M.H., 1984, Ph.D. Thesis, Univ. Toronto, Canada

Phelps R.L., Janes K.A., 1993, AJ 106, 1870

Sagar R., 1993, Current Sci. 64, 293

Sagar R., Bhatt, H.C., 1989a, BASI 17, 1

Sagar R., Bhatt, H.C., 1989b, MNRAS 236, 865

Sagar R., Cannon, R.D., 1994, BASI 22, 381

Sagar R., Cannon, R.D., 1995, A\&AS 111, 75

Sagar R., Joshi U.C., 1979, Ap\&SS 66, 3 
Sagar R., Piskunov A.E., Myakutin V.I., Joshi U.C., 1986, Stetson P.B., 1981, A.J 86, 1500 MNRAS 220, 383

Sagar R., Richtler T., 1991, A\&A 250, 324

Salpeter E.E., 1955, ApJ 121, 161

Schaltenbrand R., Tammann G.A., 1971, A\&AS 4, 265

Schmidt E.G., 1980, AJ 85, 158

Schmidt-Kaler Th., 1982, In: Landolt/Bornstein, Numerical Data and Functional Relationship in Science and Technology, New series, Group VI, Vol. 2b, Scaifers K. \& Voigt H.H. (eds.). Springer-Verlag, Berlin, p. 14

Schneider H., 1987, A\&AS 71, 531

Stetson P.B., 1987, PASP 99, 191

Stetson P.B., 1992, IAU col. 136 on stellar photometry - current techniques and future developments, Butler C.J. \& Elliott I. (eds.) p. 291

Turner D.G., 1982, PASP 94, 1003

Turner D.G., 1986, AJ 92, 111

Walker A.R., 1985, MNRAS 213, 889

Walker A.R., 1987, MNRAS 229, 31

Wesselink A.J., 1969, MNRAS 146, 329 